\title{
VECTOR-VALUED SINGULAR INTEGRAL OPERATORS ON MORREY TYPE SPACES AND VARIABLE TRIEBEL-LIZORKIN-MORREY SPACES
}

\begin{abstract}
Kwok-Pun Ho
The Hong Kong Institute of Education, Department of Mathematics and Information Technology 10, Lo Ping Road, Tai Po, Hong Kong, China; vkpho@ied.edu.hk

Abstract. A criteria on the vector-valued Banach function spaces $\mathcal{X}(\mathcal{B})$ is obtained so that whenever a vector-valued singular integral operator is bounded on $\mathcal{X}(\mathcal{B})$, it can be extended to be a bounded linear operator on the corresponding Morrey type spaces. Using this result, we define the generalized Triebel-Lizorkin-Morrey spaces and obtain the atomic and molecular decompositions. As a particular example of the generalized Triebel-Lizorkin-Morrey spaces, we introduce and study the variable Triebel-Lizorkin-Morrey spaces.
\end{abstract}

\section{Introduction}

The main result of this paper is the boundedness of vector-valued singular integral operators on weighted vector-valued Morrey spaces (see Definition 2.5). More precisely, we find that whenever a vector-valued singular integral operator is bounded on a Banach function space satisfying some mild conditions (see Definition 2.6), then this singular integral operator can be extended to be a bounded operator on the corresponding weighted vector-valued Morrey spaces. We call this result as the lifting principle for the weighted vector-valued Morrey spaces. The introduction of the lifting principle is motivated by the boundedness of the maximal operator on the Morrey spaces in [12] and the boundedness of singular integral operator on the Morrey spaces in [56]. For the statement and the proof of the lifting principle, see Theorem 3.1.

The notion of Morrey spaces is introduced in [54] to study the solutions of quasilinear elliptic differential equations by using their gradients. In [51, 52, 72], a family of function spaces arising from combining the Littlewood-Paley characterization and Morrey spaces is used to study the solutions of some non-linear differential equations, in particular, the Navier-Stokes system in Morrey spaces [29, 40, 51]. The above result also inspires a substantial amount of researches on function spaces. For instance, using the ideas from the definition of Triebel-Lizorkin spaces [24, 73] and the boundedness of the maximal operator on Morrey spaces [12, 71], Sawano, Tanaka, Tang, Wang and $\mathrm{Xu}[66,71,74]$ prove that the Triebel-Lizorkin-Morrey spaces (in [71, 74], they are called as the Morrey type Besov-Triebel spaces) are well-defined and possess the atomic and molecular decompositions. Actually, it gives an affirmative answer to a conjecture proposed by Mazzucato in [52].

doi:10.5186/aasfm.2012.3746

2010 Mathematics Subject Classification: Primary 42B20, 42B25, 42B35; Secondary 46E30, $46 \mathrm{E} 40$.

Key words: Vector-valued singular integral operators, Morrey spaces, Triebel-Lizorkin-Morrey spaces, variable exponent analysis. 
In this paper, we consider an extended version of Morrey spaces, we called it the weighted vector-valued Morrey spaces (see Definition 2.5). The formulation of the weighted vector-valued Morrey spaces consists of four components. The first one is a general Banach function space. It plays the role of the Lebesgue spaces $L^{p}$ in the definition of Morrey spaces. The introduction of the weighted vector-valued Morrey spaces by using a general Banach function space is motivated by the results obtained in [32]. In [32], we find that the study of Triebel-Lizorkin spaces can be generalized by replacing the Lebesgue spaces $L^{p}$ and the sequence spaces $l^{q}$ by rearrangementinvariant Banach function spaces (r.-i.B.f.s.) and sequence spaces with UMD property (see $[9,10])$, respectively.

To generalize the results in [32] to the weighted vector-valued Morrey spaces, two main features of r.-i.B.f.s. $X$ are essential. The first one is the Hölder equality $|E|=\left\|\chi_{E}\right\|_{X}\left\|\chi_{E}\right\|_{X^{\prime}}$ where $E$ is a Lebesgue measurable set with $|E|<\infty$ and $X^{\prime}$ is the associated space of $X$ (see [7, Chapter 2, Theorem 5.2]). The second one is the notion of Boyd's indices (see [7, Chapter 3, Definition 5.12]).

Even though the Boyd indices are not necessarily well-defined and the Hölder equality does not necessarily hold on a general Banach space, we obtain some useful extensions for these two properties of r.-i.B.f.s. We introduce the notion of tempered Banach function space in Definition 2.6 so that a generalized reverse Hölder inequality is obtained for the family $\mathbf{B}=\left\{B\left(x_{0}, r\right): x_{0} \in \mathbf{R}^{n}, r>0\right\}$ (see Proposition 2.4).

The main application of the notion of Boyd's indices is on the mapping property of the dilation operator $\left(D_{t} f\right)(x)=f(t x), t>0$. For tempered B.f.s, we just have a similar mapping property for the characteristic function of $B \in \mathbf{B}$ only (see Proposition 2.5). This is definitely weaker than the result for r.-i.B.f.s. but this weaker result is already strong enough to establish the lifting principle.

The second component for the weighted vector-valued Morrey spaces is a family of sequence spaces (see Definition 2.2). The family of sequence spaces $\left\{l_{\nu}^{q(x)}\right\}_{x \in \mathbf{R}^{n}}$ given in [20] prompts the introduction of the family of sequence spaces used in this paper.

The underlying measure for defining the weighted vector-valued Morrey spaces is the weighted Lebesgue measures given by the Muckenhoupt $A_{p}$ weight functions. In order to identify the correct family of Banach function spaces associated with an $A_{p}$-weighted Lebesgue measure, we are led to the notion of $p$-convexity. We find that when $\omega \in A_{p}$, the family of $p$-convex Banach function spaces shares some important features of the weighted Lebesgue spaces $L^{p}(\omega)$ that are crucial on extending the boundedness of singular integral operators for B.f.s. to the corresponding weighted vector-valued Morrey spaces.

The final component is a "Morrey weight function". In the Morrey spaces, it is given by $|B|^{\frac{1}{p}-\frac{1}{q}}, B \in \mathbf{B}, 1<p \leq q<\infty$. As the underlying measure is a weighted Lebesgue measure, we introduce the corresponding Morrey weight functions adapted to weighted Lebesgue measures in Definition 2.4.

We apply the lifting principle to study the "generalized Triebel-Lizorkin-Morrey spaces" defined via the Littlewood-Paley function (see Definition 5.2). We find that whenever the generalized Triebel-Lizorkin-Morrey spaces are well defined relies on the boundedness of a particular singular integral operator. Furthermore, we also 
show that once that particular singular integral operator is bounded, then the generalized Triebel-Lizorkin-Morrey spaces admit the atomic and molecular decompositions. This extends a line of researches on the atomic and molecular decompositions of several families of function spaces such as the Triebel-Lizorkin spaces [24,73], the Triebel-Lizorkin-Morrey spaces [52, 66, 74], the Littlewood-Paley spaces [32], the Triebel-Lizorkin spaces $F_{p(\cdot), q(\cdot)}^{\alpha(\cdot)}\left(\mathbf{R}^{n}\right)$ and the variable Triebel-Lizorkin spaces introduced in $[20,75]$.

In order to demonstrate the use of the above results, we consider variable Morrey spaces and variable Triebel-Lizorkin-Morrey spaces. The variable exponent analysis recently gains a lot of attentions $[4,5,14,15,17,18,19,20,21,36,41,44,49,50,57$, $75]$ because the boundedness of several important linear operators in analysis, especially, the Hardy-Littlewood maximal operator, can be established for the variable Lebesgue spaces $[14,17,19]$. In addition, it provides a theoretical background on the studies of electrorheological fluid dynamics $[1,2,61,63,64]$ and imaging processing [11].

The variable exponent Morrey spaces is introduced in $[4,41]$. An extension of Triebel-Lizorkin spaces with the Lebesgue spaces replaced by the variable Lebesgue spaces is given in [20, 75]. In this paper, we obtain a similar extension for Morrey spaces and Triebel-Lizorkin-Morrey spaces and this extension is achieved by using the lifting principle.

Section 2 presents some definitions introduced in this paper, including the notion of tempered Banach function spaces and the weighted vector-valued Morrey spaces. The main result is stated and proved in Section 3. For the rest of this paper, they are applications of the lifting principle. In Section 4, we have the application of the lifting principle on the Littlewood-Paley characterization of function spaces. Section 5 gives a general study on the generalized Triebel-Lizorkin-Morrey spaces. Section 6 delivers the results for an important example of the generalized Triebel-Lizorkin-Morrey spaces, the variable Triebel-Lizorkin-Morrey spaces.

\section{Preliminarily results}

We give some definitions and preliminarily results in this section.

Let $\mathcal{S}^{\prime}\left(\mathbf{R}^{n}\right)$ denote the class of tempered distributions and $\mathcal{S}\left(\mathbf{R}^{n}\right)$ denote the class of Schwartz functions. Moreover, $\mathcal{S}_{0}\left(\mathbf{R}^{n}\right)=\left\{f \in \mathcal{S}\left(\mathbf{R}^{n}\right): \int_{\mathbf{R}^{n}} x^{\gamma} f(x) d x=\right.$ $\left.0, \forall \gamma \in \mathbf{N}^{n}\right\}$. For any $x \in \mathbf{R}^{n}$ and $r>0$, let $B(x, r)=\left\{y \in \mathbf{R}^{n}:|x-y|<r\right\}$ and $\mathbf{B}=\left\{B\left(x_{0}, r\right): x_{0} \in \mathbf{R}^{n}, r>0\right\}$.

We adopt the definition of quasi-Banach function spaces from [7, 58].

Definition 2.1. Let $\omega$ be a measure on $\mathbf{R}^{n}$. A quasi-Banach lattice $\mathcal{X} \subset$ $\mathcal{M}(\omega, \mathbf{C})$ is said to be a quasi-Banach function space (q-B.f.s.) if

(1) $\|\cdot\|_{\mathcal{X}}$ is a quasi-norm,

(2) $|f(x)| \leq|g(x)|$ a.e. on $\left(\mathbf{R}^{n}, \omega\right)$ and $g \in \mathcal{X}$ then $f \in \mathcal{X}$ and $\|f\|_{\mathcal{X}} \leq\|g\|_{\mathcal{X}}$,

(3) $0 \leq f_{i} \uparrow f$ implies $\left\|f_{i}\right\|_{\mathcal{X}} \uparrow\|f\|_{\mathcal{X}}$

(4) for any $\omega$-measurable set $E$ with $\omega(E)<\infty, \chi_{E} \in \mathcal{X}$.

If $\|\cdot\|_{\mathcal{X}}$ is a norm, $\mathcal{X}$ is a Banach space and for any $\omega$-measurable set $E$ with $\omega(E)<\infty, \int_{E} f d \omega \leq C_{E}\|f\|_{\mathcal{X}}$ for some $C_{E}>0$, then $\mathcal{X}$ is called a Banach function space (B.f.s.). 
The following definition is inspired by the family of sequence spaces $\left\{l_{\nu}^{q(x)}\right\}_{x \in \mathbf{R}^{n}}$ introduced in [20, p. 1737].

Definition 2.2. A family of Banach lattices $\mathcal{B}=\{\mathcal{B}(x)\}_{x \in \mathbf{R}^{n}}$ is called a family of variable Banach sequence spaces (v.B.s.) if $\mathcal{B}(x) \subset\left\{\left\{a_{i}\right\}_{i \in \mathbf{Z}}: a_{i} \in \mathbf{C}\right\}$ and there exists a constant $C>0$ independent of $x \in \mathbf{R}^{n}$ such that for any $k \in \mathbf{Z}$,

$$
\left|a_{k}\right| \leq C\left\|\left\{a_{i}\right\}_{i \in \mathbf{Z}}\right\|_{\mathcal{B}(x)}, \quad \forall x \in \mathbf{R}^{n} .
$$

For any v.B.s. $\mathcal{B}=\{\mathcal{B}(x)\}_{x \in \mathbf{R}^{n}}$, denote the class of $\mathcal{B}$-valued $\omega$-measurable functions by $\mathcal{M}(\omega, \mathcal{B})$. More precisely,

$$
\mathcal{M}(\omega, \mathcal{B})=\left\{f=\left\{f_{i}\right\}_{i \in \mathbf{Z}}: f_{i}(x) \text { and }\|f(x)\|_{\mathcal{B}(x)} \text { are } \omega \text {-measurable }\right\} .
$$

Let $\mathcal{B}$ be a v.B.s. and $\omega$ be a measure on $\mathbf{R}^{n}$. Suppose that $\mathcal{X}$ is a q-B.f.s. on $\left(\mathbf{R}^{n}, \omega\right)$, define

$$
\mathcal{X}(\mathcal{B})=\left\{f \in \mathcal{M}(\omega, \mathcal{B}):\|\| f(x)\left\|_{\mathcal{B}(x)}\right\|_{\mathcal{X}}<\infty\right\}
$$

and write

$$
\|f\|_{\mathcal{X}(\mathcal{B})}=\|\| f(x)\left\|_{\mathcal{B}(x)}\right\|_{\mathcal{X}} .
$$

We recall the well known definition of doubling measure from [13]. A measure $\omega$ on $\mathbf{R}^{n}$ is said to be doubling if there exists a constant $C>0$ such that

$$
\omega(B(x, 2 r)) \leq C \omega(B(x, r)), \quad \forall x \in \mathbf{R}^{n}, r>0 .
$$

We state the definition of Muckenhoupt weight functions.

Definition 2.3. For $1<p<\infty$, a locally integrable function $\omega: \mathbf{R}^{n} \rightarrow[0, \infty)$ is said to be an $A_{p}$ weight if

$$
\sup _{B \in \mathcal{B}}\left(\frac{1}{|B|} \int_{B} \omega(x) d x\right)\left(\frac{1}{|B|} \int_{B} \omega(x)^{-\frac{p^{\prime}}{p}} d x\right)^{\frac{p}{p^{\prime}}}<\infty
$$

where $p^{\prime}=\frac{p}{p-1}$. A locally integrable function $\omega: \mathbf{R}^{n} \rightarrow[0, \infty)$ is said to be an $A_{1}$ weight if

$$
\frac{1}{|B|} \int_{B} \omega(y) d y \leq C \omega(x), \text { a.e. } x \in B
$$

for some constant $C>0$. We define $A_{\infty}=\cup_{p \geq 1} A_{p}$.

For any $\omega \in A_{\infty}$ and any Lebesgue measurable set $E$, write $\omega(E)=\int_{E} \omega(x) d x$. We have the following characterizations of $A_{\infty}$ and $A_{p}$ weights.

Theorem 2.1. A locally integrable function $\omega: \mathbf{R}^{n} \rightarrow[0, \infty)$ belongs to $A_{\infty}$ if and only if there exist an $\delta_{\omega}>0$ and a constant $C_{0}>0$ such that for any $B \in \mathcal{B}$ and all measurable subsets $E$ of $B$, we have

$$
\frac{\omega(E)}{\omega(B)} \leq C_{0}\left(\frac{|E|}{|B|}\right)^{\delta_{\omega}}
$$

Proposition 2.2. If $\omega \in A_{p}$, then there exists a constant $C>0$ such that for any $x \in \mathbf{R}^{n}, r>0$ and $\lambda>1$

$$
\omega(B(x, \lambda r)) \leq C \lambda^{n p} \omega(B(x, r)) .
$$


For the proof of the preceding results, the reader is referred to [30, Theorem 9.3.3(d) and Proposition 9.1.5(9)].

We recall the notion of $p$-th power $\left(\frac{1}{p}\right.$-convexification). For any $0<p<\infty$ and any B.f.s. $\mathcal{X}$, define the $p$-th power of $\mathcal{X}, \mathcal{X}^{p}$ by

$$
f \in \mathcal{X}^{p} \Longleftrightarrow|f|^{1 / p} \in \mathcal{X}
$$

and $\mathcal{X}^{p}$ is equipped with the quasi-norm $\|f\|_{\mathcal{X}^{p}}=\left\||f|^{1 / p}\right\|_{\mathcal{X}}^{p}$. The reader is referred to [58, Section 2.2] for a complete discussion on the notion of $p$-power of quasi-Banach function space. For $0<p \leq 1, \mathcal{X}^{p}$ is a B.f.s. (see [27, Proposition 1.11]) while for $1<p<\infty$, it is a q-B.f.s. (see [58, Chapter 2, Proposition 2.22]).

We need the notion of $p$-convexity to identify the appropriate B.f.s. on $\left(\mathbf{R}^{n}, \omega\right)$ with $\omega \in A_{p}$.

Let $0<p<\infty$. A q-B.f.s. $\mathcal{X}$ is said to be $p$-convex if there exists a constant $C>0$ such that

$$
\left\|\left(\sum_{i=1}^{n}\left|f_{i}\right|^{p}\right)^{1 / p}\right\|_{\mathcal{X}} \leq C\left(\sum_{i=1}^{n}\left\|f_{i}\right\|_{\mathcal{X}}^{p}\right)^{1 / p}
$$

for any $\left\{f_{i}\right\}_{i=1}^{n} \subset \mathcal{X}$.

The notion of $p$-convexity $1 \leq p<\infty$ for Banach lattices was introduced in [45]. For the extension of the notion of $p$-convexity to quasi-Banach space, the reader is referred to [16, p. 156]. The following proposition gives a procedure to obtain an equivalent norm for a $p$-convex q-B.f.s.

Proposition 2.3. Let $1 \leq p<\infty$. If the $q$-B.f.s. $\mathcal{X}$ is $p$-convex, then

$$
\eta_{[p]}(f)=\inf \left\{\sum_{i=1}^{n}\left\|f_{i}\right\|_{\mathcal{X}^{p}}:|f| \leq \sum_{i=1}^{n}\left|f_{i}\right|, f_{i} \in \mathcal{X}^{p}, i=1,2, \ldots, n, n \in \mathbf{N}\right\}
$$

is a lattice norm and is equivalent to $\|\cdot\|_{\mathcal{X}^{p}}$. Hence, $\mathcal{X}$ admits

$$
\eta(f)=\left(\eta_{[p]}\left(|f|^{p}\right)\right)^{1 / p}
$$

as an equivalent lattice norm.

The proof of the above proposition is given by [48, Volume II, p. 54] and [58, Proposition 2.23]. We find that whenever $\mathcal{X}$ is $p$-convex, we can consider $\mathcal{X}$ as the $\frac{1}{p}$-th power of the B.f.s. generated by the norm $\eta_{[p]}$. Therefore, it gives an access to incorporate the Hölder inequality and the norm $\eta_{[p]}$ into the estimate of singular integral operators. For detail, the reader is referred to the proof of Theorem 3.1.

The final component for constructing the weighted vector-valued Morrey spaces is the family of Morrey weight functions given in the following definition.

Definition 2.4. Let $0<q \leq \infty$ and $\omega$ be a measure on $\mathbf{R}^{n}$. A Lebesgue measurable function $u(x, r): \mathbf{R}^{n} \times(0, \infty) \rightarrow(0, \infty)$ is said to be a Morrey weight function if there exist a $0 \leq \lambda<\frac{1}{q}$ and constants $C_{1}, C_{2}>0$ so that for any $x \in \mathbf{R}^{n}$, $u(x, r)>C_{1}, r \geq 1$,

$$
\begin{aligned}
\frac{u(x, 2 r)}{u(x, r)} & \leq\left(\frac{\omega(B(x, 2 r))}{\omega(B(x, r))}\right)^{\lambda}, \quad r>0, \\
C_{2}^{-1} & \leq \frac{u(x, t)}{u(x, r)} \leq C_{2}, \quad 0<r \leq t \leq 2 r .
\end{aligned}
$$

We denote the class of Morrey weight functions by $\mathcal{W}_{q, \omega}$. 
If $\omega \equiv 1$, then we write $\mathcal{W}_{q, \omega}$ by $\mathcal{W}_{q}$. In particular, $\mathcal{W}_{1}$ contains the weighted function $u(x, r)=r^{\lambda(x)}$ where $0 \leq \lambda(x)<n$ given in [4, Section 4] for the study of the variable Morrey spaces on bounded open sets of $\mathbf{R}^{n}$. Moreover, the above weight function is also used in [35] to study the weighted Hardy-Morrey spaces.

We are now ready to define one of the main families of function spaces considered in this paper, the weighted vector-valued Morrey spaces.

Definition 2.5. Let $q>0, \omega$ be a doubling measure and $\mathcal{B}$ be a v.B.s. Suppose that $\mathcal{X}$ is a q-B.f.s. on $\left(\mathbf{R}^{n}, \omega\right)$ and $u \in \mathcal{W}_{q, \omega}$. The weighted vector-valued Morrey space $\mathcal{M}_{\omega, u}^{\mathcal{X}}(\mathcal{B})$ is the collection of all $f \in \mathcal{M}(\omega, \mathcal{B})$ satisfying

$$
\|f\|_{\mathcal{M}_{\omega, u}^{\mathcal{X}}(\mathcal{B})}=\sup _{z \in \mathbf{R}^{n}, R>0} \frac{1}{u(z, R)}\left\|\chi_{B(z, R)} f\right\|_{\mathcal{X}(\mathcal{B})}<\infty .
$$

Let $\mathcal{B}(x)=\mathbf{C}, \forall x \in \mathbf{R}^{n}$. We write $\mathcal{M}_{\omega, u}^{\mathcal{X}}(\mathbf{C})=\mathcal{M}_{\omega, u}^{\mathcal{X}}$. In case $\mathcal{X}=L^{p}(\omega)$, $1 \leq p<\infty$, we write $\mathcal{M}_{\omega, u}^{\mathcal{X}}$ by $\mathcal{M}_{\omega, u}^{p}$. Furthermore, when $\omega \equiv 1$, we further simplify the notation of $\mathcal{M}_{\omega, u}^{p}$ by $\mathcal{M}_{u}^{p}$.

If $\omega$ is a weight function, $u(x, r)=\omega(B(x, r))^{\kappa}, 0<\kappa<1$ and $\mathcal{X}=L^{p}(\omega)$, then $\mathcal{M}_{\omega, u}^{\mathcal{X}}$ is the weighted Morrey spaces considered in [43]. When $\omega \equiv 1$ and $\mathcal{X}$ is a r.-i.B.f.s., $\mathcal{M}_{\omega, u}^{\mathcal{X}}$ is the Morrey-type spaces associated with r.-i.B.f.s. defined in [32]. The weighted vector-valued Morrey spaces also include the variable exponent Morrey spaces given in [41, Definition 1.2] when $u(x, r)=r^{\lambda(x)}, 0 \leq \lambda(x) \leq n$ and $\mathcal{X}$ is the variable Lebesgue space $L^{p(\cdot)}\left(\mathbf{R}^{n}\right)$.

In [20, Lemma 6.3, Theorem 3.11 and Lemma 7.1] and [24, Theorem 3.3], a special technique, so called the "r-trick", is used to study the function spaces in [20, 24]. In fact, the notion of $p$-th power is an extension of the r-trick from Lebesgue spaces to B.f.s. The reader is referred to Section 5 for the details.

For any q-B.f.s. $\mathcal{X}$, we denote the associate space (the Köthe dual) of $\mathcal{X}$ by $\mathcal{X}^{\prime}$ (see [58, p.35]). We introduce an important notion used in this paper.

Definition 2.6. Let $\omega$ be a doubling measure on $\mathbf{R}^{n}$. A q-B.f.s. $\mathcal{X}$ on $\left(\mathbf{R}^{n}, \omega\right)$ is called tempered if the Hardy-Littlewood maximal operator with respect to $\omega$

$$
\left(\mathrm{M}_{\omega} f\right)(x)=\sup _{r>0} \frac{1}{\omega(B(x, r))} \int_{B(x, r)}|f(y)| d \omega
$$

is bounded on $\left(\mathcal{X}^{\prime}\right)^{q}$ for some $q>1$. We define the supreme of those $q>1$ such that the maximal operator is bounded on $\left(\mathcal{X}^{\prime}\right)^{q}$ to be $e_{\mathcal{X}}^{\prime}$ and let the exponent of $\mathcal{X}, e_{\mathcal{X}}$, be the conjugate of $e_{\mathcal{X}}^{\prime}$. That is, $\frac{1}{e_{\mathcal{X}}}+\frac{1}{e_{\mathcal{X}}^{\prime}}=1$.

When $\mathcal{X}$ is a rearrangement-invariant (r.-i.) q-B.f.s., the exponent of $\mathcal{X}$ is the upper Boyd index of $\mathcal{X}$ (see [55, Theorem 5] for quasi-Banach spaces and [7, Chapter 3, Definition 5.12 and Theorem 5.17] for Banach spaces). So, the notion of exponent can be considered as an extension of the notion of Boyd's indices to non-rearrangementinvariant q-B.f.s. The reader is also referred to [34] for a generalization of the Boyd indices to B.f.s and some of its applications such as the Fefferman-Stein vector-valued maximal inequalities and the Littlewood-Paley characterization of B.f.s.

Since the boundedness of $\mathrm{M}_{\omega}$ on $\left(\mathcal{X}^{\prime}\right)^{q}$ is equivalent to the boundedness of

$$
\left(\mathrm{M}_{\omega}^{q} f\right)(x)=\sup _{r>0}\left(\frac{1}{\omega(B(x, r))} \int_{B(x, r)}|f(y)|^{q} d \omega\right)^{1 / q}
$$


on $\mathcal{X}^{\prime}$. Using Jensen's inequality, we have $\mathrm{M}_{\omega}^{r} f \leq \mathrm{M}_{\omega}^{q} f$ when $1 \leq r \leq q$. As $\left\|\left(\mathrm{M}_{\omega}^{r} f\right)\right\|_{\mathcal{X}^{\prime}}^{r}=\left\|\mathrm{M}_{\omega}\left(|f|^{r}\right)\right\|_{\left(\mathcal{X}^{\prime}\right)^{r}}, \mathrm{M}_{\omega}^{r}$ is bounded on $\mathcal{X}^{\prime}$ when $1 \leq r<e_{\mathcal{X}}^{\prime}$. In particular, $\mathrm{M}_{\omega}$ is bounded on $\mathcal{X}^{\prime}$. This fact is used to obtain the following proposition.

Proposition 2.4. Let $\omega$ be a doubling measure. If $\mathcal{X}$ is a tempered B.f.s. on $\left(\mathbf{R}^{n}, \omega\right)$, then we have a constant $C>0$ so that for any $B \in \mathbf{B}$,

$$
\omega(B) \leq\left\|\chi_{B}\right\|_{\mathcal{X}}\left\|\chi_{B}\right\|_{\mathcal{X}^{\prime}} \leq C \omega(B) .
$$

Proof. The first inequality follows from the definition of associate space. For the second inequality, we consider the projection operator $P_{B}(g), B=B\left(x_{0}, r\right), x_{0} \in \mathbf{R}^{n}$ and $r>0$, defined by

$$
\left(P_{B} g\right)(y)=\left(\frac{1}{\omega(B)} \int_{B}|g| d \omega\right) \chi_{B}(y)
$$

As $\omega$ is a doubling measure, $P_{B}$ is uniformly dominated by the maximal operator $\mathrm{M}_{\omega}$. Precisely, there exists a constant $C>0$ such that for any $B=B\left(x_{0}, r\right)$, $P_{B}(f) \leq C \mathrm{M}_{\omega}(f)$. Hence, $\sup _{B}\left\|P_{B}\right\|_{\mathcal{X}^{\prime} \rightarrow \mathcal{X}^{\prime}}<C\left\|\mathrm{M}_{\omega}\right\|_{\mathcal{X}^{\prime} \rightarrow \mathcal{X}^{\prime}}$ where $\|\cdot\| \mathcal{X}_{\mathcal{X}^{\prime} \rightarrow \mathcal{X}^{\prime}}$ denote the operator norm of mapping on $\mathcal{X}^{\prime}$.

The uniformly boundedness of $P_{B}$ and [7, Chapter 1, Theorem 2.9] ensure that

$$
\left\|\chi_{B}\right\|_{\mathcal{X}}\left\|\chi_{B}\right\|_{\mathcal{X}^{\prime}}=\sup \left\{\left|\int_{B} g d \omega\right|\left\|\chi_{B}\right\|_{\mathcal{X}^{\prime}}: g \in \mathcal{X}^{\prime},\|g\|_{\mathcal{X}^{\prime}} \leq 1\right\} \leq C \omega(B) .
$$

Proposition 2.5. Let $\omega$ be a doubling measure. If $\mathcal{X}$ is a tempered B.f.s. on $\left(\mathbf{R}^{n}, \omega\right)$, then for any $1 \leq q<e_{\mathcal{X}}^{\prime}$, there exists a constant $C>0$ such that for any $x_{0} \in \mathbf{R}^{n}$ and $r>0$, we have

$$
\frac{\left\|\chi_{B\left(x_{0}, 2^{j} r\right)}\right\|_{\mathcal{X}^{\prime}}}{\left\|\chi_{B\left(x_{0}, r\right)}\right\|_{\mathcal{X}^{\prime}}} \leq C\left(\frac{\omega\left(B\left(x_{0}, 2^{j} r\right)\right)}{\omega\left(B\left(x_{0}, r\right)\right)}\right)^{\frac{1}{q}}, \quad \forall j \in \mathbf{N}
$$

and

$$
\frac{\left\|\chi_{B\left(x_{0}, r\right)}\right\|_{\mathcal{X}}}{\left\|\chi_{B\left(x_{0}, 2^{j} r\right)}\right\|_{\mathcal{X}}} \leq C\left(\frac{\omega\left(B\left(x_{0}, r\right)\right)}{\omega\left(B\left(x_{0}, 2^{j} r\right)\right)}\right)^{1-\frac{1}{q}}, \quad \forall j \in \mathbf{N} .
$$

Proof. Since $\omega$ is a doubling measure, for any $B=B\left(x_{0}, r\right) \in \mathbf{B}$ and $j \in \mathbf{N}$, we have a constant $C>0$ such that

$$
C \frac{\omega\left(B\left(x_{0}, r\right)\right)}{\omega\left(B\left(x_{0}, 2^{j} r\right)\right)} \leq \mathrm{M}_{\omega}\left(\chi_{B}\right)(x)
$$

when $x \in B\left(x_{0}, 2^{j} r\right), j \in \mathbf{N}$. Thus,

$$
\frac{\omega\left(B\left(x_{0}, r\right)\right)}{\omega\left(B\left(x_{0}, 2^{j} r\right)\right)}\left\|\chi_{B\left(x_{0}, 2^{j} r\right)}\right\|_{\left(\mathcal{X}^{\prime}\right)^{q}} \leq C\left\|\mathrm{M}_{\omega}\left(\chi_{B}\right)\right\|_{\left(\mathcal{X}^{\prime}\right)^{q}} \leq C\left\|\chi_{B}\right\|_{\left(\mathcal{X}^{\prime}\right)^{q} .} .
$$

Inequality (2.6) follows from the above inequalities. Finally, Proposition 2.4 and (2.6) yield (2.7).

The results in Propositions 2.4 and 2.5 for r.-i.q-B.f.s. are given in [33].

On one hand, the above estimate plays a decisive role on the establishment of the main result for the boundedness of singular integral operators on the weighted vector-valued Morrey spaces. On the other hand, it also justifies the introduction of the notion of exponent. Even though inequalities (2.6)-(2.7) are no longer valid for 
a general $f \in \mathcal{X}$, the estimate of the characteristic function of $B(x, r)$ based on the characteristic function of $B\left(x, 2^{j} r\right)$ is sufficient to obtain our main result.

The subsequent proposition shows that $\mathcal{M}_{\omega, u}^{\mathcal{X}}$ contains the collection of simple functions if $\omega$ belongs to $A_{\infty}$. Thus, in the following sections, we only pay our attention to those weighted vector-valued Morrey spaces associated with $A_{\infty}$-weighted Lebesgue measures.

Proposition 2.6. Let $1 \leq p<\infty$ and $\omega \in A_{\infty}$. If $\mathcal{X}^{p}$ is a tempered B.f.s. and $u \in \mathcal{W}_{p e_{\mathcal{X}}, \omega}$, then $\chi_{E} \in \mathcal{M}_{\omega, u}^{\mathcal{X}}$ for any $\omega$-measurable bounded set $E$.

Proof. Let $E$ be a $\omega$-measurable bounded set with $E \subseteq B(0, R), R>0$. For any $x_{0} \in \mathbf{R}^{n}$ and $k \in \mathbf{Z}$, write $D_{k}=B\left(x_{0}, 2^{-k}\right)$. When $k \in \mathbf{N}, D_{k} \cap E \neq \emptyset$ only if $D_{0} \subset B(0, R+2)$. Thus, Theorem 2.1, inequality (2.7) and the definition of $\mathcal{W}_{\text {pe }_{\mathcal{X}}, \omega}$ offer a $q>0$ satisfying $1 \leq q<e_{\mathcal{X}^{p}}^{\prime}$ and $\lambda<\frac{1}{p}\left(1-\frac{1}{q}\right)$ so that

$$
\frac{\left\|\chi_{D_{k} \cap E}\right\|_{\mathcal{X}}}{u\left(x_{0}, 2^{-k}\right)} \leq \frac{\left\|\chi_{D_{k}}\right\|_{\mathcal{X}^{p}}^{\frac{1}{p}}}{u\left(x_{0}, 2^{-k}\right)} \leq C \frac{\left\|\chi_{D_{0}}\right\|_{\mathcal{X}^{p}}^{\frac{1}{p}}}{u\left(x_{0}, 1\right)}\left(\frac{\omega\left(B\left(x_{0}, 2^{-k}\right)\right)}{\omega\left(B\left(x_{0}, 1\right)\right)}\right)^{\frac{1}{p}\left(1-\frac{1}{q}\right)-\lambda} \leq C\left\|\chi_{B(0, R+2)}\right\|_{\mathcal{X}}
$$

for some constant $C$ independent of $k$ and $x_{0}$. If $k<0$, then

$$
\frac{\left\|\chi_{D_{k} \cap E}\right\|_{\mathcal{X}}}{u\left(x_{0}, 2^{-k}\right)} \leq \frac{\left\|\chi_{E}\right\|_{\mathcal{X}}}{u\left(x_{0}, 2^{-k}\right)} \leq C
$$

for some $C>0$ independent of $k$. As $u$ satisfies (2.5), the above inequalities assure that $\chi_{E} \in \mathcal{M}_{\omega, u}^{\mathcal{X}}$.

We show the applications of Proposition 2.6 in Lemma 4.1 for the LittlewoodPaley characterization of function spaces and in Lemma 5.3 for the convergence of the $\psi$ transform.

\section{The lifting principle}

The study of linear operators on Morrey spaces using interpolation is obtained in $[59,60,68,69,70]$. Using the idea from the boundedness of the Hardy-Littlewood maximal operator on Morrey spaces [12], the boundedness of the singular integral operators on Morrey spaces is obtained in [53, 56].

The investigation of the vector-valued singular integral operators is started in [6]. The reader is referred to $[8,9,10,28,62]$ for the development of the theory of vector-valued singular integral operators.

One of the main results of this paper is the boundedness of singular integral operators on weighted vector-valued Morrey spaces. We begin with the definition of v.B.s.-valued singular integral operators.

Definition 3.1. Let $\omega \in A_{\infty}, \mathcal{B}_{1}=\left\{\mathcal{B}_{1}(x)\right\}$ and $\mathcal{B}_{2}=\left\{\mathcal{B}_{2}(x)\right\}$ be v.B.s. A linear operator $T: \mathcal{M}\left(\omega, \mathcal{B}_{1}\right) \rightarrow \mathcal{M}\left(\omega, \mathcal{B}_{2}\right)$ is called a v.B.s.-valued singular integral operator if there exists $K(x, y): \mathcal{B}_{1}(y) \rightarrow \mathcal{B}_{2}(x)$ such that

$$
(T f)(x)=\int K(x, y) f(y) d y, \quad \forall x \in \mathbf{R}^{n} \backslash \operatorname{supp} f
$$

and

$$
\|K(x, y)\|_{\mathcal{B}_{1}(y) \rightarrow \mathcal{B}_{2}(x)} \leq C|x-y|^{-n}, \quad \forall(x, y) \in \mathbf{R}^{2 n} \backslash\left\{(x, x): x \in \mathbf{R}^{n}\right\}
$$

for some $C>0$. We call $K(x, y)$ the kernel of $T$. 
In order to simplify the presentation, we introduce the following notations.

Definition 3.2. Let $1 \leq p<\infty$. We say that $(\omega, u, \mathcal{X})$ belongs to the class $\mathbf{M}_{p}$ if $\omega \in A_{p}, \mathcal{X}$ is a $p$-convex B.f.s. on $\left(\mathbf{R}^{n}, \omega\right), \mathcal{X}^{p}$ is a tempered B.f.s. and $u \in \mathcal{W}_{p_{\mathcal{X}} p, \omega}$.

According to Proposition 2.3, when $\mathcal{X}$ is $p$-convex for some $1 \leq p<\infty, \mathcal{X}^{p}$ possesses an equivalent norm $\eta_{[p]}$. Thus, $\left(\mathcal{X}^{p}, \eta_{[p]}\right)$ is a Banach function space. The associated space of $\mathcal{X}^{p}$ is nontrivial (see [7, Chapter 1, Theorem 2.2]) and, hence, the notion of tempered Banach function space for $\mathcal{X}^{p}$ is well-defined.

Let $p>1$ and $\omega \in A_{p}$. By the openness property of the $A_{p}$ class, $A_{p}=\cup_{1<r<p} A_{r}$, we have a $r<p$ such that $\omega \in A_{r}$. Therefore, for any $u \in \mathcal{W}_{p, \omega}$, we have $\left(\omega, u, L^{p}(\omega)\right) \in \mathbf{M}_{r}$.

The following is our main result. It is inspired by the results in [56, Theorems 1 and 2] and some similar results are obtained in [65]. It shows that any v.B.s.-valued singular integral operator which is bounded on the B.f.s. can be defined and, moreover, is bounded on the corresponding weighted vector-valued Morrey spaces.

Theorem 3.1. (The Lifting Principle) Let $0<s \leq 1$ and $1 \leq p<\infty$. If $(\omega, u, \mathcal{X}) \in \mathbf{M}_{p}$ and $\mathcal{B}_{1}=\left\{\mathcal{B}_{1}(x)\right\}$ and $\mathcal{B}_{2}=\left\{\mathcal{B}_{2}(x)\right\}$ are v.B.s., then a bounded v.B.s.-valued singular integral operator $T: \mathcal{X}^{s}\left(\mathcal{B}_{1}\right) \rightarrow \mathcal{X}^{s}\left(\mathcal{B}_{2}\right)$ can be extended to be a bounded operator from $\mathcal{M}_{\omega, u^{s}}^{\mathcal{X}^{s}}\left(\mathcal{B}_{1}\right)$ to $\mathcal{M}_{\omega, u^{s}}^{\mathcal{X}^{s}}\left(\mathcal{B}_{2}\right)$.

Proof. Let $f \in \mathcal{M}_{\omega, u^{s}}^{\mathcal{X}^{s}}\left(\mathcal{B}_{1}\right)$. For any $x_{0} \in \mathbf{R}^{n}$ and $r>0$, define $f_{1}=f \chi_{B\left(x_{0}, 2 r\right)}$ and $f_{2}=f\left(1-\chi_{B\left(x_{0}, 2 r\right)}\right)=f-f_{1}$. As $T$ is bounded from $\mathcal{X}^{s}\left(\mathcal{B}_{1}\right)$ to $\mathcal{X}^{s}\left(\mathcal{B}_{2}\right)$, we assert that

$$
\left\|\left(\chi_{B\left(x_{0}, r\right)}\right)\left(T f_{1}\right)\right\|_{\mathcal{X}^{s}\left(\mathcal{B}_{2}\right)} \leq C\left\|f_{1}\right\|_{\mathcal{X}^{s}\left(\mathcal{B}_{1}\right)} \leq C\left(u\left(x_{0}, 2 r\right)\right)^{s}\|f\|_{\mathcal{M}_{\omega, u^{s}}^{\mathcal{X}^{s}}\left(\mathcal{B}_{1}\right)} .
$$

Since $u$ satisfies (2.5), taking supreme over $x_{0} \in \mathbf{R}^{n}$ and $r>0$, we find that

$$
\left\|T f_{1}\right\|_{\mathcal{M}_{\omega, u}^{\mathcal{X}^{s}}\left(\mathcal{B}_{2}\right)} \leq C\|f\|_{\mathcal{M}_{\omega, u^{s}}^{\mathcal{X}^{s}}\left(\mathcal{B}_{1}\right)} .
$$

Therefore, it remains to consider $f_{2}$. For any $x \in B\left(x_{0}, r\right)$, we have

$$
T f_{2}(x)=\int K(x, y) f_{2}(y) d y
$$

because $x \in \mathbf{R}^{2 n} \backslash \operatorname{supp} f_{2}$. That is,

$$
\left\|T f_{2}(x)\right\|_{\mathcal{B}_{2}(x)} \leq C \int_{\mathbf{R}^{n} \backslash B\left(x_{0}, 2 r\right)} \frac{\left\|f_{2}(y)\right\|_{\mathcal{B}_{1}(y)}}{|x-y|^{n}} d y .
$$

Write $B_{k}=B\left(x_{0}, 2^{k} r\right)$ and define $S_{k}=B_{k+1} \backslash B_{k}$ when $k \geq 1$. For any $x \in B\left(x_{0}, r\right)$, we have

$$
|x-y|^{-n} \leq C 2^{-k n} r^{-n}, \quad \forall y \in S_{k}
$$

for some constant $C>0$. Hence, we assert that

$$
\left\|T f_{2}(x)\right\|_{\mathcal{B}_{2}(x)} \leq \frac{C}{r^{n}} \sum_{k=1}^{\infty} 2^{-k n} \int_{S_{k}}\|f(y)\|_{\mathcal{B}_{1}(y)} \frac{1}{\omega(y)} \omega(y) d y .
$$

Write $\frac{p}{s}=\theta$. As $s \leq p$, the Hölder inequality yields

$$
\int_{S_{k}}\|f(y)\|_{\mathcal{B}_{1}(y)} \frac{1}{\omega(y)} \omega(y) d y \leq\left(\int_{S_{k}}\|f(y)\|_{\mathcal{B}_{1}(y)}^{\theta} \omega(y) d y\right)^{\frac{1}{\theta}}\left(\int_{S_{k}} \omega(y)^{-\theta^{\prime}+1} d y\right)^{\frac{1}{\theta^{\prime}}}
$$


where $\theta^{\prime}$ is the conjugate of $\theta$. The Hölder inequality on the lattice norm $\eta_{[p]}$ (see [7], Chapter 1, Theorem 2.4) gives

$$
\int_{S_{k}}\|f(y)\|_{\mathcal{B}_{1}(y)}^{\theta} \omega(y) d y \leq\left\|\chi_{S_{k}}(\cdot)\right\| f(\cdot)\left\|_{\mathcal{B}_{1}(\cdot)}^{\theta}\right\|_{\eta_{[p]}}\left\|\chi_{S_{k}}\right\|_{\eta_{[p]}^{\prime}}
$$

where $\eta_{[p]}^{\prime}$ is the associate norm for $\eta_{[p]}$ (see [7, Chapter 1, Definition 2.1]). Since $\eta_{[p]}$ is an equivalent norm of $\|\cdot\|_{\mathcal{X}^{p}}, \eta_{[p]}^{\prime}$ is also equivalent to $\|\cdot\|_{\left(\mathcal{X}^{p}\right)^{\prime}}$. Thus,

$$
\left(\int_{S_{k}}\|f(y)\|_{\mathcal{B}_{1}(y)}^{\theta} \omega(y) d y\right)^{\frac{1}{\theta}} \leq C\left\|\chi_{S_{k}}(\cdot)\right\| f(\cdot)\left\|_{\mathcal{B}_{1}(\cdot)}\right\|_{\mathcal{X}^{s}}\left\|\chi_{S_{k}}\right\|_{\left(\mathcal{X}^{p}\right)^{\prime}}^{\frac{1}{\theta}}
$$

By multiplying $\chi_{B\left(x_{0}, r\right)}$ on both sides of (3.1), and, then, applying the norm $\|\cdot\|_{\mathcal{X}^{s}}$ on both sides of the resulting inequality, we obtain

$$
\begin{aligned}
& \left\|\chi_{B\left(x_{0}, r\right)}(\cdot)\right\| T f_{2}(\cdot)\left\|_{\mathcal{B}_{2}(\cdot)}\right\|_{\mathcal{X}^{s}} \\
& \leq \frac{C}{r^{n}} \sum_{k=1}^{\infty} 2^{-k n}\left\|\chi_{S_{k}}(\cdot)\right\| f(\cdot)\left\|_{\mathcal{B}_{1}(\cdot)}\right\|\left\|_{\mathcal{X}^{s}}\right\| \chi_{B\left(x_{0}, r\right)}\|\|_{\mathcal{X}^{s}}\left\|\chi_{S_{k}}\right\|_{\left(\mathcal{X}^{p}\right)^{\prime}}^{\frac{1}{\theta}}\left(\int_{S_{k}} \omega(y)^{-\theta^{\prime}+1} d y\right)^{\frac{1}{\theta^{\prime}}} .
\end{aligned}
$$

For any $\lambda<1 /{\text { pe } \mathcal{X}^{p}}$, there exists a $1<q<e_{\mathcal{X}^{p}}^{\prime}$ satisfying $\lambda<\frac{1}{p}\left(1-\frac{1}{q}\right)$. Thus, the belonging $\omega \in A_{p} \subseteq A_{\theta}$ and inequality (2.7) yield

$$
\begin{aligned}
& \left\|\chi_{B\left(x_{0}, r\right)}\right\|{\mathcal{\mathcal { X } ^ { s }}}\left\|\chi_{S_{k}}\right\|_{\left(\mathcal{X}^{p}\right)^{\prime}}^{1 / \theta} \leq C\left(\frac{\omega\left(B\left(x_{0}, r\right)\right)}{\omega\left(B\left(x_{0}, 2^{k} r\right)\right)}\right)^{\frac{s}{p}\left(1-\frac{1}{q}\right)}\left\|\chi_{B_{k}}\right\|_{\mathcal{X}^{p}}^{\frac{s}{p}}\left\|\chi_{B_{k}}\right\|_{\left(\mathcal{X}^{p}\right)^{\prime}}^{\frac{1}{\theta}} \\
& \leq C\left(\frac{\omega\left(B\left(x_{0}, r\right)\right)}{\omega\left(B\left(x_{0}, 2^{k} r\right)\right)}\right)^{\frac{s}{p}\left(1-\frac{1}{q}\right)} \omega\left(B_{k}\right)^{\frac{1}{\theta}} \\
& \leq C\left(\frac{\omega\left(B\left(x_{0}, r\right)\right)}{\omega\left(B\left(x_{0}, 2^{k} r\right)\right)}\right)^{\frac{s}{p}\left(1-\frac{1}{q}\right)}\left|B_{k}\right|\left(\int_{B_{k}} \omega(y)^{-\theta^{\prime}+1} d y\right)^{-\frac{1}{\theta^{\prime}}} .
\end{aligned}
$$

Thus,

$$
\begin{aligned}
& \frac{1}{\left(u\left(x_{0}, r\right)\right)^{s}}\left\|\chi_{B\left(x_{0}, r\right)}(\cdot)\right\| T f_{2}(\cdot)\left\|_{\mathcal{B}_{2}(\cdot)}\right\|_{\mathcal{X}^{s}} \\
& \leq C \sum_{k=0}^{\infty}\left(\frac{\omega\left(B\left(x_{0}, r\right)\right)}{\omega\left(B\left(x_{0}, 2^{k} r\right)\right)}\right)^{\frac{s}{p}\left(1-\frac{1}{q}\right)} \frac{1}{\left(u\left(x_{0}, r\right)\right)^{s}}\left\|\chi_{S_{k}}(\cdot)\right\| f(\cdot)\left\|_{\mathcal{B}_{1}(\cdot)}\right\|_{\mathcal{X}^{s}} \\
& \leq C \sum_{k=0}^{\infty}\left(\frac{\omega\left(B\left(x_{0}, r\right)\right)}{\omega\left(B\left(x_{0}, 2^{k} r\right)\right)}\right)^{\frac{s}{p}\left(1-\frac{1}{q}\right)}\left(\frac{u\left(x_{0}, 2^{k} r\right)}{u\left(x_{0}, r\right)}\right)^{s}\|f\|_{\mathcal{M}_{\omega, u^{s}}^{\mathcal{X}}\left(\mathcal{B}_{1}\right)} \\
& \leq C \sum_{k=0}^{\infty} 2^{k n s \delta_{\omega}\left(-\frac{1}{p}\left(1-\frac{1}{q}\right)+\lambda\right)}\|f\|_{\mathcal{M}_{\omega, u^{s}}^{\mathcal{X}^{s}}\left(\mathcal{B}_{1}\right)}
\end{aligned}
$$

where we used (2.2) for the last inequality.

As $0 \leq \lambda<\frac{1}{p}\left(1-\frac{1}{q}\right)$ and the constant $C>0$ on the above inequalities is independent of $x_{0}$ and $r$, the lifting principle is established by taking supreme over $x_{0} \in \mathbf{R}^{n}$ and $r>0$ on the above inequalities.

In order to show that the generalized Triebel-Lizorkin-Morrey spaces are welldefined, we need to apply the lifting principle to a family of singular integral operators on $\mathcal{X}^{s}, 0<s \leq 1$. This is the reason why we consider the function spaces $\mathcal{X}^{s}$, $0<s \leq 1$, in the lifting principle instead of $\mathcal{X}$. Especially, when $\mathcal{X}$ is a Lebesgue 
space, the above technique becomes the r-trick. The reader is referred to Section 5 for the details.

Here are some straightforward applications of the preceding theorem. The following result is an application of Theorem 3.1 to [48, Volume II, Theorem 1.f.14] (see also [45]).

Corollary 3.2. Let $(\omega, u, \mathcal{X}) \in \mathbf{M}_{p}$. Suppose that $T: \mathcal{X} \rightarrow \mathcal{X}$ is a bounded singular integral operator. Then, there is a constant $C>0$ such that for any $\left\{f_{i}\right\}_{i=0}^{\infty} \subset \mathcal{M}_{\omega, u}^{\mathcal{X}}$

$$
\left\|\left(\sum_{i=0}^{\infty}\left|T f_{i}\right|^{2}\right)^{1 / 2}\right\|_{\mathcal{M}_{\omega, u}^{\mathcal{X}}} \leq C\left\|\left(\sum_{i=0}^{\infty}\left|f_{i}\right|^{2}\right)^{1 / 2}\right\|_{\mathcal{M}_{\omega, u}^{\mathcal{X}}} .
$$

Similarly, we have the subsequent vector-valued inequalities on Morrey spaces.

Corollary 3.3. Let $1<p, r<\infty$ and $u \in \mathcal{W}_{p}$. If $\left\{T_{i}\right\}_{i \in \mathbf{N}}$ are a family convolution operators with their kernels $\left\{K_{i}(x)\right\}_{i \in \mathbf{N}}$ satisfying the Hörmander condition

$$
\int_{|x|>2|y|} \sup _{i}\left|K_{i}(x-y)-K_{i}(x)\right| d x \leq C
$$

for some constant $C>0$, then we have

$$
\left\|\left(\sum_{i=0}^{\infty}\left|T_{i} f_{i}\right|^{r}\right)^{1 / r}\right\|_{\mathcal{M}_{u}^{p}} \leq C\left\|\left(\sum_{i=0}^{\infty}\left|f_{i}\right|^{r}\right)^{1 / r}\right\|_{\mathcal{M}_{u}^{p}} .
$$

The lifting principle gives us an access to investigate the weighted vector-valued Morrey space through the results from the corresponding vector-valued Banach function spaces. For the rest of this paper, we use the lifting principle to introduce and study some new Morrey type spaces related to several Banach function spaces appeared in $[4,5,20,24,25,26,32,35,41,42,43,44,47,52,56,66,67,71,73,74,75]$.

We present the Littlewood-Paley characterization of Morrey type space in the subsequent section. We use the lifting principle to introduce and study a generalization of Triebel-Lizorkin-Morrey space in Section 5. Finally, we apply the results in Section 5 to study the Morrey space with variable exponent in Sections 6 .

\section{Littlewood-Paley characterization}

As the first "non-straightforward" application of the lifting principle, we consider the Littlewood-Paley characterization of function spaces. In this section, we extract the conditions imposed on the B.f.s. $\mathcal{X}$ so that the corresponding weighted vectorvalued Morrey spaces $\mathcal{M}_{\omega, u}^{\mathcal{X}}$ possess the Littlewood-Paley characterization.

We begin with the definition of Littlewood-Paley characterization. For any $\varphi \in$ $\mathcal{S}_{0}\left(\mathbf{R}^{n}\right)$ satisfying

$$
\operatorname{supp} \hat{\varphi} \subset\left\{\xi \in \mathbf{R}^{n}: 1 / 2 \leq|\xi| \leq 2\right\}, \quad \text { and } \quad|\hat{\varphi}(\xi)| \geq C, \quad 3 / 5 \leq|\xi| \leq 5 / 3
$$

for some $C>0$, define $\varphi_{j}(x)=2^{j n} \varphi\left(2^{j} x\right), j \in \mathbf{Z}$.

Definition 4.1. For any B.f.s. $\mathcal{X}$ satisfying $\mathcal{S}_{0}\left(\mathbf{R}^{n}\right) \hookrightarrow \mathcal{X} \hookrightarrow \mathcal{S}^{\prime}\left(\mathbf{R}^{n}\right) / \mathcal{P}$ where $\mathcal{P}$ is the class of polynomials on $\mathbf{R}^{n}$, we say that $\mathcal{X}$ possesses the Littlewood-Paley 
characterization if we have the Banach space isomorphism $\mathcal{X}=\dot{F}_{l^{2}, \mathcal{X}}^{0}$ where

$$
\dot{F}_{l^{2}, \mathcal{X}}^{0}=\left\{f \in \mathcal{S}^{\prime}\left(\mathbf{R}^{n}\right) / \mathcal{P}:\|f\|_{\dot{F}_{l^{2}, \mathcal{X}}^{0}}=\left\|\left(\sum_{j \in \mathbf{Z}}\left|f * \varphi_{j}\right|^{2}\right)^{1 / 2}\right\|_{\mathcal{X}}<\infty\right\}
$$

and $\|\cdot\|_{\dot{F}_{l^{2}, \mathcal{X}}^{0}}$ is an equivalent norm of $\|\cdot\|_{\mathcal{X}}$.

Define $\mathcal{G}_{\varphi}$ by

$$
\mathcal{G}_{\varphi}(f)=\left\{f * \varphi_{j}\right\}_{j \in \mathbf{Z}}, \quad \forall f \in \mathcal{S}^{\prime}\left(\mathbf{R}^{n}\right) / \mathcal{P} .
$$

Note that a necessary condition for $\mathcal{X}$ possessing the Littlewood-Paley characterization is the boundedness of the operator $\mathcal{G}_{\varphi}: \mathcal{X} \rightarrow \mathcal{X}\left(l^{2}\right)$. In addition, a sufficient condition for $\mathcal{X}$ having the Littlewood-Paley characterization is the boundedness of the operators $\mathcal{G}_{\varphi}$ and its adjoint $\mathcal{G}_{\varphi}^{*}: \mathcal{X}\left(l^{2}\right) \rightarrow \mathcal{X}$. Let $\varphi, \psi \in \mathcal{S}_{0}\left(\mathbf{R}^{n}\right)$ satisfy (4.1) and $\sum_{j \in \mathbf{Z}} \hat{\varphi}\left(2^{-j} \xi\right) \overline{\hat{\psi}\left(2^{-j} \xi\right)}=1, \xi \neq 0$ where $\hat{\varphi}$ and $\hat{\psi}$ are the Fourier transforms of $\varphi$ and $\psi$, respectively. Using the Littlewood-Paley analysis [31], the adjoint operator of $\mathcal{G}_{\varphi}$ (with respect to the Lebesgue measure) is defined by

$$
\mathcal{G}_{\varphi}^{*}\left(\left\{f_{j}\right\}_{j \in \mathbf{Z}}\right)=\sum_{j \in \mathbf{Z}} f_{j} * \psi_{j} .
$$

We need to impose a stronger condition on the B.f.s. $\mathcal{X}$ in order to overcome a technical obstacle on establishing the Littlewood-Paley characterization of $\mathcal{M}_{\omega, u}^{\mathcal{X}}$.

Definition 4.2. We say that a B.f.s. $\mathcal{X}$ on $\left(\mathbf{R}^{n}, \omega\right)$ is of polynomial growth if there exist a $\beta>0$ and a constant $C>0$ so that for any $x_{0} \in \mathbf{R}^{n}$ and $r \geq 1$, we have $\left\|\chi_{B\left(x_{0}, r\right)}\right\|_{\mathcal{X}} \leq C \omega\left(B\left(x_{0}, r\right)\right)^{\beta}$.

We write $(\omega, u, \mathcal{X}) \in \mathbf{P}_{p}$ if $\mathcal{X}$ is of polynomial growth and $(\omega, u, \mathcal{X}) \in \mathbf{M}_{p}$.

For example, according to (2.6), if $\mathcal{X}$ is a tempered B.f.s., then $\mathcal{X}^{\prime}$ is of polynomial growth. Furthermore, as long as the maximal operator is bounded on $\mathcal{X}^{q}$ for some $0<q<\infty, \mathcal{X}$ is of polynomial growth. The proof of this assertion follows from the proof of Proposition 2.5. For brevity, we leave the proof to the reader.

Lemma 4.1. Let $1 \leq p<\infty$. If $(\omega, u, \mathcal{X}) \in \mathbf{P}_{p}$, then $\mathcal{S}\left(\mathbf{R}^{n}\right) \hookrightarrow \mathcal{M}_{\omega, u}^{\mathcal{X}} \hookrightarrow$ $\mathcal{S}^{\prime}\left(\mathbf{R}^{n}\right) / \mathcal{P}$.

Proof. From the proof of Proposition 2.6, for any $r>0$, we have

$$
\left\|\chi_{B(0, r)}\right\|_{\mathcal{M}_{\omega, u}^{\mathcal{X}}} \leq C \sup _{|x| \leq 1+r}\left(\left\|\chi_{B(x, 1)}\right\|_{\mathcal{X}},\left\|\chi_{B(0, r)}\right\|_{\mathcal{X}}\right) \leq C \omega(B(0,2+r))^{\beta} .
$$

Since $\omega \in A_{p}$, for $M>n p \beta+1$, we have a constant $C_{M}>0$ such that for any $\varphi \in \mathcal{S}\left(\mathbf{R}^{n}\right)$

$$
\|\varphi\|_{\mathcal{M}_{\omega, u}^{\mathcal{X}}} \leq \sum_{j \in \mathbf{N}} 2^{-M j}\left\|\chi_{B\left(0,2^{j}\right)}\right\|_{\mathcal{M}_{\omega, u}^{\mathcal{X}}} \leq C \sum_{j \in \mathbf{N}} 2^{-M j} 2^{(j+1) n p \beta}<\infty .
$$

Thus, $\mathcal{S}\left(\mathbf{R}^{n}\right) \hookrightarrow \mathcal{M}_{\omega, u}^{\mathcal{X}}$.

From (3.2)-(3.4), we find that for any $\varphi \in \mathcal{S}^{\prime}\left(\mathbf{R}^{n}\right)$ and $0<\alpha<1$, there exists a constant $C>0$ such that

$$
\left|\int f(x) \varphi(x) d x\right| \leq C \sum_{k=0}^{\infty} 2^{-k(n+\alpha)}\left\|\chi_{S_{k}} f\right\|_{\mathcal{X}}\left\|\chi_{S_{k}}\right\|_{\left(\mathcal{X}^{p}\right)^{\prime}}^{\frac{1}{p}}\left(\int_{S_{k}} \omega(y)^{-p^{\prime}+1} d y\right)^{\frac{1}{p^{\prime}}}
$$

where $S_{0}=B(0,2)$ and $S_{k}=B\left(0,2^{k+1}\right) \backslash B\left(0,2^{k}\right), k \in \mathbf{N}$. 
Then, (3.5)-(3.6) guarantee that $f \in \mathcal{S}^{\prime}\left(\mathbf{R}^{n}\right)$ with order zero where we use the notion of order for Schwartz distribution introduced in [31]. Furthermore, according to [31, Theorem 3.1], $\mathcal{M}_{\omega, u}^{\mathcal{X}} \cap \mathcal{P}$ only contains constant function. So, it suffices to show that $F \equiv 1$ does not belong to $\mathcal{M}_{\omega, u}^{\mathcal{X}}$ because $\mathcal{M}_{\omega, u}^{\mathcal{X}}$ is a lattice. We prove by contradiction. Assuming that $F \in \mathcal{M}_{\omega, u}^{\mathcal{X}}$. According to the definition of $\mathcal{W}_{p_{e_{\mathcal{X}}}, \omega}$, we have a $0<\lambda<\frac{1}{p e_{\mathcal{X}} p}$ so that

$$
\frac{u\left(0,2^{k}\right)}{u(0,1)} \leq\left(\frac{\omega\left(B\left(0,2^{k}\right)\right)}{\omega(B(0,1))}\right)^{\lambda}, \quad \forall k \in \mathbf{N} .
$$

Thus, we have a $q$ satisfying $1<q<e_{\mathcal{X}^{p}}^{\prime}$ and $0<\lambda<\frac{1}{p}\left(1-\frac{1}{q}\right)$. For any $k \in \mathbf{N}$, inequality (2.7) guarantees that

$$
\left(\frac{\omega\left(B\left(0,2^{k}\right)\right)}{\omega(B(0,1))}\right)^{\frac{1}{p}\left(1-\frac{1}{q}\right)} \frac{\left\|\chi_{B(0,1)}\right\|_{\mathcal{X}}}{u\left(0,2^{k}\right)} \leq C \frac{\left\|\chi_{B\left(0,2^{k}\right)}\right\|_{\mathcal{X}}}{u\left(0,2^{k}\right)} \leq C\left\|\chi_{F}\right\|_{\mathcal{M}_{\omega, u}^{\mathcal{X}}} .
$$

Subsequently,

$$
\left(\frac{\omega\left(B\left(0,2^{k}\right)\right)}{\omega(B(0,1))}\right)^{\frac{1}{p}\left(1-\frac{1}{q}\right)} \leq C \frac{u\left(0,2^{k}\right)}{u(0,1)} \leq C\left(\frac{\omega\left(B\left(0,2^{k}\right)\right)}{\omega(B(0,1))}\right)^{\lambda} .
$$

Since $\omega\left(B\left(0,2^{k}\right)\right) \rightarrow \infty$ as $k \rightarrow \infty$, the above inequality contradicts the fact that $0<\lambda<\frac{1}{p}\left(1-\frac{1}{q}\right)$. Thus, we conclude that $\mathcal{M}_{\omega, u}^{\mathcal{X}} \cap \mathcal{P}=\{0\}$.

We now state the result for the Littlewood-Paley characterization of $\mathcal{M}_{\omega, u}^{\mathcal{X}}$.

Theorem 4.2. Let $1 \leq p<\infty$ and $(\omega, u, \mathcal{X}) \in \mathbf{P}_{p}$. If $\mathcal{G}_{\varphi}$ is bounded from $\mathcal{X}$ to $\mathcal{X}\left(l^{2}\right)$ and $\mathcal{G}_{\varphi}^{*}$ is bounded from $\mathcal{X}\left(l^{2}\right)$ to $\mathcal{X}$, then $\mathcal{M}_{\omega, u}^{\mathcal{X}}$ possesses the Littlewood-Paley characterization.

Proof. The kernel of the operator $\mathcal{G}_{\varphi}, K(x, y)=\left\{\varphi_{j}(x-y)\right\}_{j \in \mathbf{Z}}$ satisfies for any $m \in \mathbf{N}$, there exists a $C>0$ such that

$$
\left(\sum_{j \in \mathbf{Z}}\left|\varphi_{j}(x-y)\right|^{2}\right)^{\frac{1}{2}} \leq C\left(\sum_{j \in \mathbf{Z}} \frac{2^{2 j n}}{\left(1+2^{j}|x-y|\right)^{2 m}}\right)^{\frac{1}{2}} \leq C|x-y|^{-n},
$$

for all $x, y \in \mathbf{R}^{n}, x \neq y$. Thus, Theorem 3.1 and the boundedness of $\mathcal{G}_{\varphi}$ on $\mathcal{X}$ establish the embedding $\mathcal{M}_{\omega, u}^{\mathcal{X}} \hookrightarrow \dot{F}_{l^{2}, \mathcal{M}_{\omega, u}^{\mathcal{X}}}^{0}$ and the inequality $\|f\|_{\dot{F}_{l^{2}, \mathcal{M}_{\omega, u}^{\mathcal{X}}}^{0}} \leq C\|f\|_{\mathcal{M}_{\omega, u}^{\mathcal{X}}}$.

Similarly, as the kernel of $\mathcal{G}_{\varphi}^{*}$ also satisfies (4.2), Theorem 3.1 asserts that

$$
\left\|\mathcal{G}_{\varphi}^{*}\left(\left\{f_{j}\right\}_{j \in \mathbf{Z}}\right)\right\|_{\mathcal{M}_{\omega, u}^{\mathcal{X}}} \leq C\left\|\left(\sum_{j \in \mathbf{Z}}\left|f_{j}\right|^{2}\right)^{1 / 2}\right\|_{\mathcal{M}_{\omega, u}^{\mathcal{X}}} .
$$

Hence, letting $f_{j}=f * \varphi_{j}$, we have

$$
\left\|\sum_{j \in \mathbf{Z}} f * \varphi_{j} * \psi_{j}\right\|_{\mathcal{M}_{\omega, u}^{\mathcal{X}}} \leq C\|f\|_{\dot{F}_{l^{2}, \mathcal{M} \omega, u}^{\mathcal{X}}} .
$$

In view of the Littlewood-Paley analysis [31] and the fact that $\mathcal{M}_{\omega, u}^{\mathcal{X}} \cap \mathcal{P}=\{0\}$, the embedding $\dot{F}_{l^{2}, \mathcal{M}_{\omega, u}^{\mathcal{X}}}^{\mathcal{X}} \hookrightarrow \mathcal{M}_{\omega, u}^{\mathcal{X}}$ holds.

For the sake of illustrating the use of Theorem 4.2, we consider two examples. 
In the first example, we consider the case $\omega \equiv 1$. The boundedness of the operator $\mathcal{G}_{\varphi}^{*}: \mathcal{X}\left(l^{2}\right) \rightarrow \mathcal{X}$ can be obtained from the boundedness of $\mathcal{G}_{\varphi}: \mathcal{X}^{\prime} \rightarrow \mathcal{X}^{\prime}\left(l^{2}\right)$ via the Lorentz-Luxemburg theorem (see [7, Chapter 1, Theorem 2.7]). More precisely, we have the following corollary. For simplicity, we leave the proof of the subsequent corollary to the reader.

Corollary 4.3. Let $(|\cdot|, u, \mathcal{X}) \in \mathbf{P}_{1}$. If $\mathcal{G}_{\varphi}$ is bounded from $\mathcal{X}$ to $\mathcal{X}\left(l^{2}\right)$ and from $\mathcal{X}^{\prime}$ to $\mathcal{X}^{\prime}\left(l^{2}\right)$, then $\mathcal{M}_{u}^{\mathcal{X}}$ possesses the Littlewood-Paley characterization.

Let $(|\cdot|, u, \mathcal{X}) \in \mathbf{P}_{1}$ and $\left(|\cdot|, v, \mathcal{X}^{\prime}\right) \in \mathbf{P}_{1}$. If both $\mathcal{X}$ and $\mathcal{X}^{\prime}$ possess the Littlewood-Paley characterization, then $\mathcal{M}_{v}^{\mathcal{X}}$ and $\mathcal{M}_{u}^{\mathcal{X}^{\prime}}$ also possess the Littlewood-Paley characterization. In particular, if $\mathcal{X}$ is r.-i. with Boyd's indices strictly bigger than one and strictly less than infinity, then $\mathcal{M}_{u}^{\mathcal{X}}$ and $\mathcal{M}_{v}^{\mathcal{X}^{\prime}}$ possess the Littlewood-Paley characterization (see [32, Theorem 5.6]).

We consider the Morrey spaces generated by weighted Lebesgue spaces $L^{p}(\omega)$ associated with $A_{p}$ weights as the second example.

Corollary 4.4. Let $1<p<\infty$ and $\omega \in A_{p}$. If $u \in \mathcal{W}_{p, \omega}$, then $\mathcal{M}_{\omega, u}^{p}$ possesses the Littlewood-Paley characterization.

Proof. Obviously, we have $\left(\omega, u, L^{p}(\omega)\right) \in \mathbf{P}_{r}$ for some $0<r<p$. The boundedness of $\mathcal{G}_{\varphi}$ from $L^{p}(\omega)$ to $L^{p}(\omega)\left(l^{2}\right)$ follows from the proof of [47, Theorem 3.1]. For the boundedness of $\mathcal{G}_{\varphi}^{*}$, we find that

$$
\begin{aligned}
& \left\|\mathcal{G}_{\varphi}^{*}\left(\left\{f_{j}\right\}_{j \in \mathbf{Z}}\right)\right\|_{L^{p}(\omega)} \\
& =\sup _{\|g\|_{L^{p^{\prime}}(\omega)} \leq 1}\left|\int \sum_{j \in \mathbf{Z}}\left(f_{j} * \psi_{j}\right)(x) g(x) \omega(x) d x\right| \\
& \left.=\sup _{\|g\|_{L^{p^{\prime}(\omega)}} \leq 1} \mid \int \sum_{j \in \mathbf{Z}} f_{j}(x)\left((g \omega) * \psi_{j}\right)\right)(x) d x \mid \\
& \leq \sup _{\|g\|_{L^{p^{\prime}}(\omega)} \leq 1}\left\|\left\{f_{j}\right\}_{j \in \mathbf{Z}}\right\|_{L^{p}(\omega)\left(l^{2}\right)}\left(\int\left(\sum_{j \in \mathbf{Z}}\left|\left((g \omega) * \psi_{j}\right)(x)\right|^{2}\right)^{\frac{p^{\prime}}{2}} \omega(x)^{-\frac{p^{\prime}}{p}} d x\right)^{\frac{1}{p^{\prime}}}
\end{aligned}
$$

where $p^{\prime}$ is the conjugate of $p$. In view of the fact that $\omega^{-\frac{p^{\prime}}{p}} \in A_{p^{\prime}}$, we obtain

$$
\left(\int\left(\sum_{j \in \mathbf{Z}}\left|\left((g \omega) * \psi_{j}\right)(x)\right|^{2}\right)^{\frac{p^{\prime}}{2}} \omega(x)^{-\frac{p^{\prime}}{p}} d x\right)^{\frac{1}{p^{\prime}}} \leq C\|g \omega\|_{L^{p^{\prime}}\left(\omega^{-\frac{p^{\prime}}{p}}\right)}
$$

by using the boundedness of $\mathcal{G}_{\varphi}$ from $L^{p^{\prime}}\left(\omega^{-\frac{p^{\prime}}{p}}\right)$ to $L^{p^{\prime}}\left(\omega^{-\frac{p^{\prime}}{p}}\right)\left(l^{2}\right)$. Finally, as

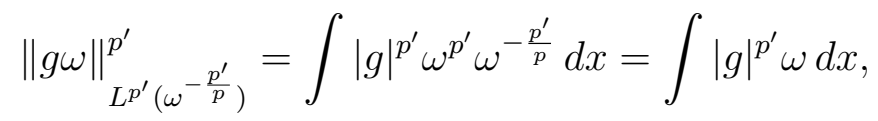

we obtain the boundedness of $\mathcal{G}_{\varphi}^{*}: L^{p}(\omega)\left(l^{2}\right) \rightarrow L^{p}(\omega)$ and, hence, our desired result follows from Theorem 4.2.

Notice that the weighted Morrey spaces given in [43, Definition 2.1] are particular cases of $\mathcal{M}_{\omega, u}^{p}$. Thus, they also admit the Littlewood-Paley characterization. 


\section{Generalized Triebel-Lizorkin-Morrey spaces}

The second application of the lifting principle is a study of a family of function spaces defined via the Littlewood-Paley function. We call them the generalized Triebel-Lizorkin-Morrey spaces.

The conjecture from [52] inspires a new direction of researches for function spaces. More precisely, the study of Triebel-Lizorkin spaces can be combined with Morrey spaces to produce the Triebel-Lizorkin-Morrey spaces [52, 66, 71, 74] which are generalizations of Triebel-Lizorkin spaces and Morrey spaces. In this section, we further extend these generalizations by introducing the generalized Triebel-LizorkinMorrey spaces.

We apply the lifting principle to show that if $\mathcal{X}$ satisfies some vector-valued inequalities, then the generalized Triebel-Lizorkin-Morrey spaces are well-defined. We extend our analysis by considering the quasi-Banach function space $\mathcal{X}^{p}, 1<p<$ $\infty$. To show that the generalized Triebel-Lizorkin-Morrey spaces associated with $\mathcal{X}^{p}$ is well-defined, it requires the validity of a family of vector-valued inequalities on $\mathcal{X}^{s}$ for $0<s \leq 1$. At the end of this section, we obtain the atomic and molecular decompositions for the generalized Triebel-Lizorkin-Morrey spaces.

We now state the definition of the generalized Triebel-Lizorkin-Morrey spaces.

Definition 5.1. Let $0<q<\infty$ and $\mathcal{B}$ be a v.B.s. Define $\mathcal{B}^{q}$ by $\mathcal{B}^{q}=$ $\left\{\mathcal{B}(x)^{q}\right\}_{x \in \mathbf{R}^{n}}$.

When $1<q<\infty, \mathcal{B}^{q}$ is a family of quasi-Banach spaces.

Definition 5.2. Let $1 \leq p, q, r<\infty,(\omega, u, \mathcal{X}) \in \mathbf{M}_{p}$ and $\mathcal{B}$ be a v.B.s. The generalized Triebel-Lizorkin-Morrey space $F_{\mathcal{M}_{\omega, u^{r}}^{\mathcal{X}^{r}}}^{\mathcal{X}^{r}}(\Phi)$ consists of those $f \in \mathcal{S}^{\prime}\left(\mathbf{R}^{n}\right)$ satisfying

$$
\|f\|_{F_{\mathcal{M}_{\omega, u^{r}}^{\mathcal{B}^{r}}}^{\mathcal{X}^{r}}(\Phi)}=\left\|\left\{f * \varphi_{j}\right\}_{j=0}^{\infty}\right\|_{\mathcal{M}_{\omega, u^{r}}^{\mathcal{X}^{r}}\left(\mathcal{B}^{q}\right)}<\infty
$$

where $\varphi_{0} \in \mathcal{S}\left(\mathbf{R}^{n}\right)$ and $\varphi_{j}(x)=2^{j n} \varphi\left(2^{j} x\right), j \geq 1, \varphi \in \mathcal{S}\left(\mathbf{R}^{n}\right)$. The pair $\Phi=\left(\varphi_{0}, \varphi\right)$ satisfies

$$
\hat{\varphi}_{0} \subset\left\{\xi \in \mathbf{R}^{n}:|\xi| \leq 1\right\} \text { and } \hat{\varphi} \subseteq\left\{\xi \in \mathbf{R}^{n}: 1 / 2 \leq|\xi| \leq 2\right\}
$$

and $\hat{\varphi}_{0}+\hat{\varphi}_{1}=1$ on $\left\{\xi \in \mathbf{R}^{n}:|\xi| \leq 1\right\}$ and $\hat{\varphi}(2 \xi)+\hat{\varphi}(\xi)+\hat{\varphi}(\xi / 2)=1$ on $\{\xi \in$ $\left.\mathbf{R}^{n}: 1 / 2 \leq|\xi| \leq 2\right\}$.

When $r=\bar{q}=1$, we write $F_{\mathcal{M}_{\omega, u^{r}}^{\mathcal{X}^{r}}}^{\mathcal{B}^{r}}$ by $F_{\mathcal{M}_{\omega, u}^{\mathcal{X}}}^{\mathcal{B}}$.

The assumptions imposed on the pair $\left(\varphi_{0}, \varphi\right)$ can be relaxed so that the Littlewood-Paley analysis is valid and the family of function spaces $F_{\mathcal{M}_{\omega, u^{r}}^{\mathcal{X}^{r}}}^{\mathcal{X}^{q}}(\Phi)$ is independent of $\left(\varphi_{0}, \varphi\right)$. For the sake of brevity, we skip the details and refer the reader to $[24,25,31]$.

The above definition encompasses several "Triebel-Lizorkin-Morrey" type spaces. Obviously, it covers the classical Triebel-Lizorkin space [73] and the function spaces studied in [20, 52, 66, 71, 74, 75]. It also includes some non-Triebel-Lizorkin-Morrey type spaces. For instance, the family of generalized Triebel-Lizorkin-Morrey spaces covers the inhomogeneous version of the Littlewood-Paley spaces associated with r.-i.q-B.f.s. introduced in [32].

In order to have a well defined definition for $F_{\mathcal{M}_{\omega, u^{r}}^{\mathcal{X}^{r}}}^{\mathcal{B}^{q}}=F_{\mathcal{M}_{\omega, u^{r}}^{\mathcal{X}^{r}}}^{\mathcal{B}^{q}}(\Phi)$, we have to show that it is independent of the functions $\varphi_{0}$ and $\varphi$ used in Definition 5.2. We 
have two methods to show that $F_{\mathcal{M}_{\omega, u^{r}}^{\mathcal{X}^{r}}}^{\mathcal{B}^{q}}$ is well defined. Both of them rely on the lifting principle given in Theorem 3.1. The following definition gives us a criteria on the v.B.s. so that the linear operators used in the subsequent theorems are singular integral operators.

Definition 5.3. We say that $\left(\omega, u, \mathcal{X}, \mathcal{B}_{1}, \mathcal{B}_{2}\right)$ belongs to $\mathbf{V}_{p}$ if $(\omega, u, \mathcal{X}) \in \mathbf{M}_{p}$ and there exist a constant $C$ independent of $x, y \in \mathbf{R}^{n}$ and a bounded non-negative function $\gamma(x, y)$ so that for any $x, y \in \mathbf{R}^{n}$ with $x \neq y$,

$$
|x-y|^{-\gamma(x, y)}\left\|\left\{2^{-\gamma(x, y) j} a_{j}\right\}_{j=0}^{\infty}\right\|_{\mathcal{B}_{2}(x)} \leq C\left\|\left\{a_{j}\right\}_{j=0}^{\infty}\right\|_{\mathcal{B}_{1}(y)} .
$$

Write $\Gamma=\sup _{x, y \in \mathbf{R}^{n}} \gamma(x, y)$.

Notice that we can take $\gamma(x, y) \equiv 0$ if and only if there exists a Banach sequence space $B$ such that $\mathcal{B}(x)=B$ for all $x \in \mathbf{R}^{n}$. Moreover, as long as we have $\left(\omega, u, \mathcal{X}, \mathcal{B}_{1}, \mathcal{B}_{2}\right) \in \mathbf{V}_{p}$, we also have $\left(\omega, u, \mathcal{X}, \mathcal{B}_{1}^{q}, \mathcal{B}_{2}^{q}\right) \in \mathbf{V}_{p}$ for any $0<q<\infty$.

Lemma 5.1. Let $0<s \leq 1,1 \leq p<\infty$ and $\left(\omega, u, \mathcal{X}, \mathcal{B}_{1}, \mathcal{B}_{2}\right) \in \mathbf{V}_{p}$. Suppose that $\left\{\phi_{j}\right\}_{j=0}^{\infty} \subset \mathcal{S}\left(\mathbf{R}^{n}\right)$ satisfy

$$
\left|\phi_{j}(x)\right| \leq C 2^{n j}\left(1+2^{j}|x|\right)^{-n-\Gamma}, \quad \forall j \in \mathbf{N} \cup\{0\}
$$

for some $C>0$. If there is a constant $B>0$ so that

$$
\left\|\left\{\phi_{j} * f_{j}\right\}_{j=0}^{\infty}\right\|_{\mathcal{X}^{s}\left(\mathcal{B}_{2}\right)} \leq B\left\|\left\{f_{j}\right\}_{j=0}^{\infty}\right\|_{\mathcal{X}^{s}\left(\mathcal{B}_{1}\right)},
$$

then there exists a constant $A>0$ such that

$$
\left\|\left\{\phi_{j} * f_{j}\right\}_{j=0}^{\infty}\right\|_{\mathcal{M}_{\omega, u^{s}}^{\mathcal{X}^{s}}\left(\mathcal{B}_{2}\right)} \leq A\left\|\left\{f_{j}\right\}_{j=0}^{\infty}\right\|_{\mathcal{M}_{\omega, u^{s}}^{\mathcal{X}^{s}}\left(\mathcal{B}_{1}\right)} .
$$

Proof. Fix $x, y \in \mathbf{R}^{n}$ with $x \neq y$. Let $U_{1}(y)=\left\{a \in \mathcal{B}_{1}(y):\|a\|_{\mathcal{B}_{1}(y)} \leq 1\right\}$. The kernel of the operator $T\left(\left\{f_{j}\right\}_{j=0}^{\infty}\right)=\left\{\varphi_{j} * f_{j}\right\}_{j=0}^{\infty}, K(x, y)$, satisfies

$$
\begin{aligned}
& \|K(x, y)\|_{\mathcal{B}_{1}(y) \rightarrow \mathcal{B}_{2}(x)} \\
& \leq \sup _{\left\{a_{j}\right\}_{j=0}^{\infty} \in U_{1}(y)}\left\|\left\{\varphi_{j}(x-y) a_{j}\right\}_{j=0}^{\infty}\right\|_{\mathcal{B}_{2}(x)} \\
& \leq\left. C \sup _{\left\{a_{j}\right\}_{j=0}^{\infty} \in U_{1}(y)}\left\|\left\{\frac{2^{n j} a_{j}}{\left(1+2^{j}|x-y|\right)^{n+\gamma(x, y)}}\right\}_{j=0}^{\infty}\right\|\left\|_{\mathcal{B}_{2}(x)}\right\|\right|^{-\gamma(x, y)-n}\left\|\left\{2^{-\gamma(x, y) j} a_{j}\right\}_{j=0}^{\infty}\right\| \|_{\mathcal{B}_{2}(x)} \leq C \frac{1}{|x-y|^{n}} \\
& \leq C \sup _{\left\{a_{j}\right\}_{j=0}^{\infty} \in U_{1}(y)}|x-y|^{\infty}
\end{aligned}
$$

for some $C>0$ independent of $x, y \in \mathbf{R}^{n}$. With the above estimate, Theorem 3.1 offers us our desired result.

Definition 5.4. Let $\mathcal{B}$ be a v.B.s. We say that a linear operator $T$ is bounded uniformly on $\mathcal{B}$ if there exists a constant $C>0$ independent of $x \in \mathbf{R}^{n}$ so that $\|T a\|_{\mathcal{B}(x)} \leq C\|a\|_{\mathcal{B}(x)}$ for all $a \in \mathcal{B}$ and $x \in \mathbf{R}^{n}$.

Theorem 5.2. Let $1 \leq p<\infty$ and $(\omega, u, \mathcal{X}, \mathcal{B}, \mathcal{B}) \in \mathbf{V}_{p}$. Suppose that the B.f.s. $\mathcal{X}$ satisfies (5.3) with $s=1$ and $\mathcal{B}_{1}=\mathcal{B}_{2}=\mathcal{B}$. If the shifting operators

$$
\begin{aligned}
R\left(\left\{a_{j}\right\}_{j=0}^{\infty}\right) & =\left\{a_{j+1}\right\}_{j=0}^{\infty} \\
L\left(\left\{a_{j}\right\}_{j=0}^{\infty}\right) & =\left\{a_{j-1}\right\}_{j=0}^{\infty} \text { with } a_{-1}=0,
\end{aligned}
$$

are bounded uniformly on $\mathcal{B}$, then the function space $F_{\mathcal{M}_{\omega, u}^{\mathcal{X}}}^{\mathcal{B}}$ is well defined. 
Proof. Let $\Phi=\left(\varphi_{0}, \varphi\right)$ and $\Psi=\left(\psi_{0}, \psi\right)$ be two pairs of functions satisfy the conditions for defining the function space $F_{\mathcal{M}_{\omega, u}^{\mathcal{X}}}^{\mathcal{B}}$ in Definition 5.2. Lemma 5.1 yields

$$
\left\|\left\{\psi_{j} * f_{j}\right\}_{j=0}^{\infty}\right\|_{\mathcal{M}_{\omega, u}^{\mathcal{X}}(\mathcal{B})} \leq A\left\|\left\{f_{j}\right\}_{j=0}^{\infty}\right\|_{\mathcal{M}_{\omega, u}^{\mathcal{X}}(\mathcal{B})} .
$$

For any $f \in F_{\mathcal{M}_{w, u}^{\mathcal{X}}}^{\mathcal{B}}(\Phi)$, applying the above inequality with $f_{0}=\varphi_{0} * f+\varphi_{1} * f$ and $f_{j}=\varphi_{j-1} * f+\varphi_{j} * f+\varphi_{j+1} * f$ when $j \geq 1$, we have

$$
\begin{aligned}
\left\|\left\{\psi_{j} * f\right\}_{j=0}^{\infty}\right\|_{\mathcal{M}_{\omega, u}^{\mathcal{X}}(\mathcal{B})} & =\left\|\left\{\psi_{j} * f_{j}\right\}_{j=0}^{\infty}\right\|_{\mathcal{M}_{\omega, u}^{\mathcal{X}}(\mathcal{B})} \leq A\left\|\left\{f_{j}\right\}_{j=0}^{\infty}\right\|_{\mathcal{M}_{\omega, u}^{\mathcal{X}}(\mathcal{B})} \\
& \leq 3 A\left\|\left\{\varphi_{j} * f\right\}_{j=0}^{\infty}\right\|_{\mathcal{M}_{\omega, u}^{\mathcal{X}}(\mathcal{B})}
\end{aligned}
$$

because $\psi_{j} * f_{j}=\psi_{j} * f, j \in \mathbf{N} \cup\{0\}$ and $R$ and $L$ are bounded. The independence of the definition of the function space $F_{\mathcal{M}_{\omega, u}^{\mathcal{X}}}^{\mathcal{B}}$ from the pair of functions $\left(\varphi_{0}, \varphi\right)$ satisfying the conditions in Definition 5.2 follows from the above inequality.

We apply the above result to the weighted Lebesgue spaces. Let $1<p, q<\infty$ and $\omega \in A_{p}$. If we assign $\mathcal{X}=L^{p}(\omega)$ and $\mathcal{B}_{1}(x)=\mathcal{B}_{2}(x)=l^{q}$ for all $x \in \mathbf{R}^{n}$, then inequality (5.3) is valid for all $0<s \leq 1$ (see [3, Theorem 5.2]). Therefore, the $A_{p^{-}}$ weighted versions of the Triebel-Lizorkin-Morrey spaces considered in [66, 71, 74] are well defined.

Notice that the drawback of Theorem 5.2 is the restriction $q=r=1$. That is, the "function space" component and the "sequence space" component of $F_{\mathcal{M}_{\omega, u^{r}}^{\mathcal{X}^{r}}}^{\mathcal{B}^{q}}$ are Banach spaces. In order to study the case $1 \leq q, r<\infty$, we present the second method for showing the independence of the definition of $F_{\mathcal{M}_{\omega, u^{r}}^{\mathcal{X}^{r}}}^{\mathcal{X}^{r}}$ on the pair $\left(\varphi_{0}, \varphi\right)$. We use the $\varphi-\psi$ transform introduced by Frazier and Jawerth in [22, 23, 24, 25].

We recall the definition of the inhomogeneous $\varphi-\psi$ transforms from [24, Section 12].

Let $\mathcal{Q}=\left\{Q_{\nu, k}: \nu \in \mathbf{N} \cup\{0\}, k \in \mathbf{Z}^{n}\right\}$ where $Q_{\nu, k}=\left\{\left(x_{1}, \ldots, x_{n}\right) \in \mathbf{R}^{n}: k_{j} \leq\right.$ $\left.2^{\nu} x_{j}<k_{j}+1, j=1, \ldots, n\right\}$ and $k=\left(k_{1}, \ldots, k_{n}\right)$. Denote the lower-left corner of the dyadic cube $Q$ by $x_{Q}=2^{-\nu} k$, the side-length by $l(Q)$ and the Lebesgue measure of $Q$ by $|Q|$. Moreover, we write $\varphi_{Q}(x)=2^{n \nu} \varphi\left(2^{\nu} x-k\right)$ when $Q=Q_{\nu, k}$.

For any $f \in \mathcal{S}^{\prime}\left(\mathbf{R}^{n}\right)$, denote the Fourier transform of $f$ by $\hat{f}$. Let $\varphi_{0}, \psi_{0} \in \mathcal{S}\left(\mathbf{R}^{n}\right)$ and $\varphi, \psi \in \mathcal{S}_{0}\left(\mathbf{R}^{n}\right)$ satisfying

$$
\begin{aligned}
& \operatorname{supp} \hat{\varphi}_{0}, \operatorname{supp} \hat{\psi}_{0} \subseteq\left\{\xi \in \mathbf{R}^{n}:|\xi| \leq 2\right\}, \\
& \left|\hat{\varphi}_{0}(\xi)\right|,\left|\hat{\psi}_{0}(\xi)\right| \geq c>0, \text { if }|\xi| \leq 5 / 3, \\
& \operatorname{supp} \hat{\varphi}, \operatorname{supp} \hat{\psi} \subseteq\left\{\xi \in \mathbf{R}^{n}: 1 / 2 \leq|\xi| \leq 2\right\}, \\
& |\hat{\varphi}(\xi)|,|\hat{\psi}(\xi)| \geq c>0, \text { if } 3 / 5 \leq|\xi| \leq 5 / 3, \\
& \overline{\hat{\varphi}_{0}(\xi)} \hat{\psi}_{0}(\xi)+\sum_{\nu=1}^{\infty} \frac{\hat{\varphi}\left(2^{-\nu} \xi\right)}{\psi}\left(2^{-\nu} \xi\right)=1, \forall \xi \in \mathbf{R}^{n} .
\end{aligned}
$$

For any complex-valued sequence $s=\left\{s_{Q}\right\}_{Q \in \mathcal{Q}}$ and $f \in \mathcal{S}^{\prime}\left(\mathbf{R}^{n}\right)$, define $\mathrm{S}_{\varphi}(f)=$ $\left\{\left\langle f, \varphi_{Q}\right\rangle\right\}_{Q \in \mathcal{Q}}$ and $\mathrm{T}_{\psi}(s)=\sum_{Q \in \mathcal{Q}} s_{Q} \psi_{Q}$.

In order to study the $\varphi-\psi$ transform, we need to introduce the sequence space associated with $F_{\mathcal{M}_{\omega, u^{r}}^{\mathcal{X}^{r}}}^{\mathcal{B}^{r}}$. 
Definition 5.5. Let $1 \leq p, q, r<\infty$ and $(\omega, u, \mathcal{X}, \mathcal{B}, \mathcal{B}) \in \mathbf{V}_{p}$. The sequence space $f_{\mathcal{M}_{\omega, u^{r}}^{\mathcal{X}^{r}}}^{\mathcal{B}^{q}}$ consists of those complex-valued sequence $s=\left\{s_{Q}\right\}_{Q \in \mathcal{Q}}$ satisfying

$$
\|s\|_{f_{\mathcal{M}_{\omega, u^{\gamma}}^{\mathcal{X}^{r}}}}=\left\|\left\{\sum_{k \in \mathbf{Z}^{n}}\left|s_{Q_{j, k}}\right| \tilde{\chi}_{Q_{j, k}}\right\}_{j=0}^{\infty}\right\|_{\mathcal{M}_{\omega, u^{r}}^{\mathcal{X}^{r}}\left(\mathcal{B}^{q}\right)}<\infty
$$

where $\tilde{\chi}_{Q}=|Q|^{-1 / 2} \chi_{Q}$ and $Q \in \mathcal{Q}$.

When $q=r=1$, we write $f_{\mathcal{M}_{\omega, u^{r}}^{\mathcal{X}^{q}}}^{\mathcal{X}^{r}}$ by $f_{\mathcal{M}_{\omega, u}^{\mathcal{X}}}^{\mathcal{B}}$.

If $u \equiv 1$, we write $F_{\mathcal{M}_{\omega, u^{r}}^{\mathcal{B}^{q}}}^{\mathcal{X}^{r}}$ and $f_{\mathcal{M}_{\omega, u^{r}}^{\mathcal{X}^{r}}}^{\mathcal{X}^{q}}$ by $F_{\mathcal{X}^{r}}^{\mathcal{B}^{q}}$ and $f_{\mathcal{X}^{r}}^{\mathcal{B}^{q}}$, respectively.

To study the case $1<q, r<\infty$, it requires a stronger condition imposed on the B.f.s. and the v.B.s., we call that the admissibility condition.

We introduce some notations used to formulate the admissibility condition. Let $\eta_{m}(x)=(1+|x|)^{-m}$ and $\eta_{\nu, m}(x)=2^{n \nu} \eta_{m}\left(2^{\nu} x\right), m, \nu \in \mathbf{N} \cup\{0\}$. For any $0<\theta, \beta$ and any sequence $a=\left\{a_{j}\right\}_{j=0}^{\infty}$, define

$$
\vec{S}_{\theta}(a)=\left\{\sum_{j=0}^{i} 2^{(j-i)(\theta+\epsilon)} a_{j}\right\}_{i=0}^{\infty} \text { and } \overleftarrow{S}_{\beta}(a)=\left\{\sum_{j=i}^{\infty} 2^{(j-i)(\beta-\epsilon)} a_{j}\right\}_{i=0}^{\infty}
$$

Definition 5.6. Let $\theta, \beta>0, \mathcal{X}$ be a B.f.s. and $\mathcal{B}$ be a v.B.s. We say that $(\mathcal{X}, \mathcal{B})$ is admissible with order $(\theta, \beta)$ if

(1) there exist $M>0$ and $0<s_{0} \leq 1$ so that for any $0<s, t<s_{0}$ and $m>M$,

$$
\left\|\left\{\eta_{j, m} * f_{j}\right\}_{j=0}^{\infty}\right\|_{\mathcal{X}^{s}\left(\mathcal{B}^{t}\right)} \leq C\left\|\left\{f_{j}\right\}_{j=0}^{\infty}\right\|_{\mathcal{X}^{s}\left(\mathcal{B}^{t}\right)}
$$

for some $C>0$; and

(2) for any $\epsilon>0$, there exists $C>0$ independent of $x \in \mathbf{R}^{n}$ so that

$$
\left\|\vec{S}_{\theta}(a)\right\|_{\mathcal{B}(x)} \leq C\|a\|_{\mathcal{B}(x)} \quad \text { and } \quad\left\|\overleftarrow{S}_{\beta}(a)\right\|_{\mathcal{B}(x)} \leq C\|a\|_{\mathcal{B}(x)}
$$

Let $1 \leq p, q<\infty$. If $\mathcal{X}=L^{p}\left(\mathbf{R}^{n}\right)$ and $\mathcal{B}(x)=l^{q}, \forall x \in \mathbf{R}^{n}$, then $(\mathcal{X}, \mathcal{B})$ satisfies Condition (1) of Definition 5.6 with $s_{0}=1$ and $M=n$. Let $\alpha \geq 0$ and $1 \leq s<\infty$. When $\mathcal{B}(x)=l^{\alpha, s}, \forall x \in \mathbf{R}^{n}$ where

$$
l^{\alpha, s}=\left\{a=\left(a_{i}\right)_{i=0}^{\infty}:\|a\|_{l^{\alpha, s}}=\left(\sum_{i=0}^{\infty}\left(2^{i \alpha}\left|a_{i}\right|\right)^{s}\right)^{\frac{1}{s}}\right\},
$$

$\{\mathcal{B}(x)\}_{x \in \mathbf{R}^{n}}$ fulfills Condition (2) of Definition 5.6 with $\theta=\beta=\alpha$.

The admissibility condition also reveals the reason why we consider $\mathcal{X}^{s}$ with $0<s \leq 1$ instead of $\mathcal{X}$ in the lifting principle, Theorem 3.1.

The introduction of condition (1) in the preceding definition is motivated by [20, Theorem 3.2]. For condition (2), it is related to an obstacle arising from the definition of the $\psi$-transform. The $\psi$-transform is defined by an infinite series while the convergence of this series is not guaranteed. The subsequent result overcomes this difficulty by showing that whenever $(\mathcal{X}, \mathcal{B})$ is admissible, the series used to define the $\psi$-transform converges in $\mathcal{S}^{\prime}\left(\mathbf{R}^{n}\right)$.

Lemma 5.3. Let $1 \leq p, q, r<\infty, 0<\theta, \beta$ and $(\omega, u, \mathcal{X}, \mathcal{B}, \mathcal{B}) \in \mathbf{V}_{p}$. If $(\mathcal{X}, \mathcal{B})$ is admissible with order $(\theta, \beta)$, then the $\psi$-transform is a well-defined mapping from $f_{\mathcal{M}_{\omega, u^{r}}^{\mathcal{X}^{r}}}^{\mathcal{X}^{r}}$ to $\mathcal{S}^{\prime}\left(\mathbf{R}^{n}\right)$. 
Proof. Let $s=\left\{s_{Q}\right\}_{Q \in \mathcal{Q}} \in f_{\mathcal{M}_{\omega, u^{r}}^{\mathcal{X}^{r}}}^{\mathcal{B}^{r}}$ and $f=\sum_{Q \in \mathcal{Q}} s_{Q} \psi_{Q}$. Take $0<h<$ $\min \left(\frac{s_{0}}{r}, \frac{s_{0}}{q}\right)$. For any given $g \in \mathcal{S}\left(\mathbf{R}^{n}\right)$ and $|Q|=2^{-n \mu}, \mu \in \mathbf{N}$, according to [24, Lemmas 3.6 and B.1], we assert that for any $L>\max (M, n+\Gamma) / h$,

$$
\left|g * \psi_{Q}(x)\right| \leq C 2^{-\mu\left(L-q \beta-\frac{n}{2}\right)}\left(1+\left|x_{Q}-x\right|\right)^{-L}
$$

where $M$ is the constant given in Condition (1) of Definition 5.6.

Multiplying $s_{Q}$ on both sides of the above inequality and, then, taking summation over $Q \in \mathcal{Q}$, we obtain

$$
\sum_{Q \in \mathcal{Q}}\left|s_{Q}\right|\left|g * \psi_{Q}(x)\right| \leq C \sum_{\mu=0}^{\infty} 2^{-\mu\left(L-q \beta-\frac{n}{2}\right)} \sum_{l(Q)=2^{-\mu}}\left|s_{Q}\right| /\left(1+\left|x-x_{Q}\right|\right)^{L} .
$$

An estimate from [24, p. 50] assures that

$$
\sum_{Q \in \mathcal{Q}}\left|s_{Q}\right|\left|g * \psi_{Q}(x)\right| \leq C \sum_{\mu=0}^{\infty} 2^{\mu\left(q \beta-\frac{\epsilon}{2}\right)}\left(\left(\sum_{k \in \mathbf{Z}^{n}}\left|s_{Q_{\mu, k}}\right| \tilde{\chi}_{Q_{\mu, k}}\right)^{h} * \eta_{\mu, h L}\right)^{\frac{1}{h}}
$$

as $h L>\max (M, n+\Gamma)$. that

Write $A_{\nu}=\left(\left(\sum_{k \in \mathbf{Z}^{n}}\left|s_{Q_{\nu, k}}\right| \tilde{\chi}_{Q_{\nu, k}}\right)^{h} * \eta_{\nu, h L}\right)^{\frac{1}{h}}$. Inequalities (2.1) and (5.5) assure

$$
\left\|\sum _ { Q \in \mathcal { Q } } \left|s_{Q}\left\|g * \psi_{Q}(x) \mid\right\|_{\mathcal{M}_{\omega, u^{r}}^{\mathcal{X}^{r}}} \leq C\|\| \overleftarrow{S}_{q \beta}\left(\left\{A_{\nu}\right\}_{\nu \in \mathbf{N}}\right)\left\|_{\mathcal{B}^{q}(x)}\right\|_{\mathcal{M}_{\omega, u^{r}}^{\mathcal{X}^{r}}}\right.\right.
$$

For any $1<q$, the boundedness of $\overleftarrow{S}_{\beta}$ on $\mathcal{B}$ is equivalent to the boundedness of $\overleftarrow{S}_{q \beta}$ on $\mathcal{B}^{q}$. Thus, $\left\|\overleftarrow{S}_{q \beta}\left(\left\{A_{\nu}\right\}_{\nu \in \mathbf{N}}\right)\right\|_{\mathcal{B}^{q}(x)} \leq C\left\|\left(\left\{A_{\nu}\right\}_{\nu \in \mathbf{N}}\right)\right\|_{\mathcal{B}^{q}(x)}$ for some $C>0$ independent of $x \in \mathbf{R}^{n}$. Using Condition (1) of Definition 5.6 and Lemma 5.1, we obtain

$$
\begin{aligned}
\left\|\sum _ { Q \in \mathcal { Q } } \left|s_{Q}\left\|g * \psi_{Q}(x) \mid\right\|_{\mathcal{M}_{\omega, u^{r}}^{\mathcal{X}^{r}}}\right.\right. & \leq C\left\|\left\{A_{\nu}\right\}_{\nu \in \mathbf{N}}\right\|_{\mathcal{M}_{\omega, u^{r}}^{\mathcal{X}^{r}}\left(\mathcal{B}^{q}\right)} \\
& \leq C\left\|\left\{A_{\nu}^{h}\right\}_{\nu=0}^{\infty}\right\|_{\mathcal{M}_{\omega, u^{h r}}^{\mathcal{X}^{h r}}\left(\mathcal{B}^{h q}\right)}^{\frac{1}{h}} \leq C\|s\|_{\mathcal{F}_{\mathcal{M}_{\omega, u^{r}}^{\mathcal{X}}}^{\mathcal{X}^{r}}}<\infty .
\end{aligned}
$$

Proposition 2.6 guarantees that $\sum_{Q \in \mathcal{Q}}\left|s_{Q}\right|\left|g * \psi_{Q}\right|$ is finite almost everywhere. Therefore, $f$ is a well-defined tempered distribution.

Recall that in the lifting principle, we consider the family of function spaces $\mathcal{X}^{s}$, $0<s \leq 1$, instead of $\mathcal{X}$. As claimed at the end of Section 3, it is related to the r-trick. In fact, the admissibility condition is defined in order to incorporate the r-trick to our study of $F_{\mathcal{M}_{\omega, u^{r}} \mathcal{B}^{q}}$. The following theorem reveals in detail on how to integrate the lifting principle, the admissibility condition and the r-trick together to show the boundedness of the $\varphi-\psi$ transform.

Theorem 5.4. Let $1 \leq p, q, r<\infty$ and $(\omega, u, \mathcal{X}, \mathcal{B}, \mathcal{B}) \in \mathbf{V}_{p}$. If $(\mathcal{X}, \mathcal{B})$ is admissible, then the $\varphi$-transform $S_{\varphi}: F_{\mathcal{M}_{\omega, u^{r}}^{\mathcal{X}^{r}}}^{\mathcal{B}^{q}} \rightarrow f_{\mathcal{M}_{\omega, u^{r}}^{\mathcal{X}^{q}}}^{\mathcal{X}^{r}}$ is bounded and the $\psi$-transform $T_{\psi}: f_{\mathcal{M}_{\omega, u^{r}}^{\mathcal{X}^{q}}}^{\mathcal{X}^{q}} \rightarrow F_{\mathcal{M}_{\omega, u^{r}}^{\mathcal{X}}}^{\mathcal{B}^{q}}$ is also bounded. Hence, $F_{\mathcal{M}_{\omega, u^{r}}^{\mathcal{B}^{r}}}^{\mathcal{B}^{r}}$ is well defined. 
Proof. Take $0<h<\min \left(\frac{s_{0}}{r}, \frac{s_{0}}{q}\right)$. For any $f \in F_{\mathcal{M}_{\omega, u}^{\mathcal{X}}}^{\mathcal{B}}$, we have

$$
\left\|S_{\varphi} f\right\|_{F_{\mathcal{M}_{\omega, u^{r}}^{\mathcal{B}}}^{\mathcal{X}^{r}}}=\left\|\left\{\sum_{k \in \mathbf{Z}^{n}}\left|\left(\varphi_{\nu} * f\right)\left(x_{Q_{\nu, k}}\right)\right| \chi_{Q_{Q_{\nu, k}}}\right\}_{\nu=0}^{\infty}\right\|_{\mathcal{M}_{\omega, u^{r}}^{\mathcal{X}^{r}}\left(\mathcal{B}^{q}\right)} .
$$

Using the idea from Peetre's inequality (see [22, (2.11)] or [73, Sections 1.4.1 and 1.4.2] or [20, Lemma A.6]), for sufficiently large $m$, we find that

$$
\begin{aligned}
\left\|S_{\varphi} f\right\|_{F_{\mathcal{M}_{\omega, u^{r}}^{\mathcal{X}}}^{\mathcal{X}^{r}}} \leq C\left\|\left(\left\{\eta_{\nu, m} *\left|\varphi_{\nu} * f\right|^{h}\right)^{\frac{1}{h}}\right\}_{\nu=0}^{\infty}\right\|_{\mathcal{M}_{\omega, u^{r}}^{\mathcal{X}^{r}}\left(\mathcal{B}^{q}\right)} \\
=C\left\|\left\{\eta_{\nu, m} *\left|\varphi_{\nu} * f\right|^{h}\right\}_{\nu=0}^{\infty}\right\|_{\mathcal{M}_{\omega, u^{h r}}^{\mathcal{X}}\left(\mathcal{B}^{h q}\right)}^{\frac{1}{h}}
\end{aligned}
$$

Therefore, the admissibility condition and Lemma 5.1 conclude that

$$
\left\|S_{\varphi} f\right\|_{F_{\mathcal{M}_{\omega, u^{r}}^{\mathcal{K} q}}^{\mathcal{K}^{r}}} \leq C\left\|\left\{\left|\varphi_{\nu} * f\right|^{h}\right\}_{\nu=0}^{\infty}\right\|_{\mathcal{M}_{\omega, u^{h r}}^{\mathcal{X} h r}\left(\mathcal{B}^{h q}\right)}^{\frac{1}{h}}=C\|f\|_{F_{\mathcal{M}_{\omega, u^{r}}^{\mathcal{K}^{r}}}^{\mathcal{K}^{r}}} .
$$

We consider the $\psi$-transform. Suppose that $s=\left\{s_{Q}\right\}_{Q \in \mathcal{Q}} \in f_{\mathcal{M}_{\omega, u^{r}}^{\mathcal{X}^{r}}}^{\mathcal{X}^{q}}$ and $f=$ $T_{\psi}(s)=\sum_{Q \in \mathcal{Q}} s_{Q} \psi_{Q}$. Using Lemma 5.3, it remains to show that $f \in F_{\mathcal{M}_{\omega, u^{r}}^{\mathcal{X}^{r}}}^{\mathcal{B}^{q}}$. For any $\Phi=\left(\varphi_{0}, \varphi\right)$ satisfying the conditions in Definition 5.2, we use the estimate from $[24$, p. 50] and find that

$$
\left|\varphi_{\nu} * f\right| \leq C \sum_{\mu=\max (\nu-1,0)}^{\nu+1}\left(\left(\sum_{k \in \mathbf{Z}^{n}}\left|s_{Q_{\mu, k}}\right| \tilde{\chi}_{Q_{\mu, k}}\right)^{h} * \eta_{\mu, m}\right)^{\frac{1}{h}} .
$$

As $(\mathcal{X}, \mathcal{B})$ is admissible, $R$ and $L$ are bounded uniformly on $\mathcal{B}$. Thus, Lemma 5.1 ensures that

$$
\begin{aligned}
\left\|T_{\psi}(s)\right\|_{F_{\mathcal{M}_{\omega, u^{r}}^{\mathcal{B}}}^{\mathcal{B}}} & =\left\|\left\{f * \varphi_{\nu}\right\}_{\nu=0}^{\infty}\right\|_{\mathcal{M}_{\omega, u^{r}}^{\mathcal{X}^{r}}} \\
& \leq C\left\|\left\{\sum_{\mu=\max (\nu-1,0)}^{\nu+1}\left(\sum_{k \in \mathbf{Z}^{n}}\left|s_{Q_{\mu, k}}\right| \tilde{\chi}_{Q_{\mu, k}}\right)^{h} * \eta_{\mu, m}\right\}_{\nu=0}^{\infty}\right\|_{\mathcal{M}_{\omega, u^{h r}}^{\mathcal{X} h r}\left(\mathcal{B}^{h q}\right)}^{\frac{1}{h}} \\
& \leq C\left\|\left\{\sum_{k \in \mathbf{Z}^{n}}\left|s_{Q_{\mu, k}}\right| \tilde{\chi}_{Q_{\mu, k}}\right\}_{\nu=0}^{\infty}\right\|_{\mathcal{M}_{\omega, u^{r}}^{\mathcal{X}^{r}}\left(\mathcal{B}^{q}\right)}=C\|s\|_{f_{\mathcal{M}_{\omega, u^{r}}^{\mathcal{X}}}^{\mathcal{X}^{r}}} .
\end{aligned}
$$

With the boundedness of the $\varphi-\psi$ transform, the rest of the proof follows from some simple modifications of the arguments from [24, Theorem 2.2]. For the sake of brevity, we leave it to the reader.

The following presents another application of the $\varphi-\psi$ transform. We show that $F_{\mathcal{M}_{\omega, u^{r}}^{\mathcal{X}^{r}}}^{\mathcal{B}^{q}}$ and $f_{\mathcal{M}_{\omega, u^{r}}^{\mathcal{X}^{r}}}^{\mathcal{B}^{q}}$ are quasi-Banach spaces. We have a supporting lemma for this result. We use the techniques developed in [32, Lemma 1.6, Theorems 1.7 and 2.5] to obtain the following results.

Lemma 5.5. Let $1 \leq p, q, r<\infty$ and $(\omega, u, \mathcal{X}, \mathcal{B}, \mathcal{B}) \in \mathbf{V}_{p}$. There exists a $\rho>0$ such that for any $\left\{F_{i}\right\}_{i=0}^{\infty} \subset \mathcal{M}_{\omega, u^{r}}^{\mathcal{X}^{r}}\left(\mathcal{B}^{q}\right)$,

$$
\sum_{i=0}^{\infty}\left\|F_{i}\right\|_{\mathcal{M}_{\omega, u^{r}}^{\mathcal{X}^{r}}\left(\mathcal{B}^{q}\right)}^{\rho}<\infty \Longrightarrow \sum_{i=0}^{\infty} F_{i} \in \mathcal{M}_{\omega, u^{r}}^{\mathcal{X}^{r}}\left(\mathcal{B}^{q}\right)
$$


Proof. We find that $\|\cdot\|_{\mathcal{B}^{q}}^{1 / q}$ satisfies the triangle inequality. Since $\|\cdot\|_{\left(\mathcal{M}_{\omega, u^{r}}^{\mathcal{X}^{r}}\right)^{\frac{1}{q}}}$ is a quasi-norm, Aoki-Rolewicz theorem (see [39, Theorem 1.3]) provides a $\rho>0$ so that

$$
\left\|\left(\sum_{i=0}^{\infty}\left\|F_{i}\right\|_{\mathcal{B}^{q}}^{1 / q}\right)^{q}\right\|_{\mathcal{M}_{\omega, u^{r}}^{\mathcal{X}^{r}}} \leq C\left(\sum_{i=0}^{\infty}\left\|F_{i}\right\|_{\mathcal{M}_{\omega, u^{r}}^{\mathcal{X}^{r}}\left(\mathcal{B}^{q}\right)}^{\rho}\right)^{\frac{1}{\rho}} .
$$

As $\mathcal{X}$ is complete, the completeness of $\mathcal{M}_{\omega, u^{r}}^{\mathcal{X}^{r}}$ follows from a simple modification of the classical Morrey space (see [46, Section 4.4]). In view of the assumption $\sum_{i=0}^{\infty}\left\|F_{i}\right\|_{\mathcal{M}_{\omega, u^{r}}^{\mathcal{X}^{r}}\left(\mathcal{B}^{q}\right)}^{\rho}<\infty$ and the sub-additive of $\|\cdot\|_{\mathcal{B}^{q}}^{1 / q}$, we conclude that $\sum_{i=0}^{\infty} F_{i} \in$ $\mathcal{M}_{\omega, u^{r}}^{\mathcal{X}^{r}}\left(\mathcal{B}^{q}\right)$ and satisfies

$$
\left\|\sum_{i=0}^{\infty} F_{i}\right\|_{\left.\mathcal{M}_{\omega, u^{r}}^{\mathcal{X}^{r}} \mathcal{B}^{q}\right)} \leq C\left(\sum_{i=0}^{\infty}\left\|F_{i}\right\|_{\mathcal{M}_{\omega, u^{r}}^{\mathcal{X}^{r}}\left(\mathcal{B}^{q}\right)}^{\rho}\right)^{\frac{1}{\rho}} .
$$

Theorem 5.6. Let $1 \leq p, q, r<\infty$ and $(\omega, u, \mathcal{X}, \mathcal{B}, \mathcal{B}) \in \mathbf{V}_{p}$. Then $f_{\mathcal{M}_{\omega, u^{r}}^{\mathcal{X}^{r}}}^{\mathcal{B}^{q}}$ is a quasi-Banach space. In addition, if $(\mathcal{X}, \mathcal{B})$ is admissible, then $F_{\mathcal{M}_{\omega, u^{r}}^{\mathcal{x}^{r}}}^{\mathcal{B}^{q}}$ is also a quasi-Banach space.

Proof. Let $\rho$ be the constant given in Lemma 5.5. For any Cauchy sequence $c_{i}=\left\{c_{i, Q}\right\}_{Q \in \mathcal{Q}}, i \in \mathbf{N} \cup\{0\}$, without loss of generality, we can assume that $\sum_{i=1}^{\infty} \| c_{i}-$ $c_{i-1} \|_{f_{\mathcal{M}_{\omega, u^{\prime}}^{\mathcal{B}}}^{\mathcal{B} q}}^{\rho}<\infty$. Write

$$
C_{i}(x)=\left\{\sum_{|Q|=2^{-j n}}\left|c_{i, Q}-c_{i-1, Q}\right| \tilde{\chi}_{Q}(x)\right\}_{j=0}^{\infty}, \quad i \in \mathbf{N} .
$$

We have $\sum_{i=0}^{\infty}\left\|C_{i}\right\|_{\mathcal{M}_{\omega, u^{r}}^{\mathcal{X}^{r}}\left(\mathcal{B}^{q}\right)}^{\rho}=\sum_{i=1}^{\infty}\left\|c_{i}-c_{i-1}\right\|_{f_{\mathcal{M}_{\omega, u^{r}}^{\mathcal{X}^{r}}}^{\rho}}^{\rho}<\infty$. Thus, Lemma 5.5 assures that $C_{\infty}=\sum_{i=0}^{\infty} C_{i}$ exists in $\mathcal{M}_{\omega, u^{r}}^{\mathcal{X}^{r}}\left(\mathcal{B}^{q}\right)$. As dyadic cubes with equal Lebesgue measure are either disjoint or identical, we conclude that

$$
C_{\infty}=\left\{\sum_{|Q|=2^{-j n}}\left(\sum_{i=0}^{\infty}\left|c_{i, Q}-c_{i-1, Q}\right|\right) \tilde{\chi}_{Q}(x)\right\}_{j=0}^{\infty}, \quad i \in \mathbf{N} \cup\{0\}
$$

is well defined. Thus, for any $Q \in \mathcal{Q}, \sum_{i=1}^{\infty}\left|c_{i, Q}-c_{i-1, Q}\right|<\infty$. Indeed,

$$
c_{\infty}=\lim _{i \rightarrow \infty} c_{i}=\left\{c_{0, Q}+\sum_{i=1}^{\infty}\left(c_{i, Q}-c_{i-1, Q}\right)\right\}_{Q \in \mathcal{Q}}
$$

exists. In view of (5.6), we obtain

$$
\left\|c-c_{l}\right\|_{f_{\mathcal{M}_{\omega, u^{r}}^{\mathcal{B} q}}^{\mathcal{X}^{r}}}=\left\|C_{\infty}-\sum_{i=1}^{l} C_{i}\right\|_{\mathcal{M}_{\omega, u^{r}}^{\mathcal{X}^{r}\left(\mathcal{B}^{q}\right)}} \leq C\left(\sum_{i=l+1}^{\infty}\left\|c_{i}-c_{i-1}\right\|_{f_{\mathcal{M}_{\omega, u^{r}}^{\mathcal{X}^{r}}}^{\rho}}^{\rho}\right)^{\frac{1}{\rho}} \rightarrow 0
$$

as $i$ goes to infinity.

We now turn to the completeness of $F_{\mathcal{M}_{\omega, u^{r}}^{\mathcal{B}^{q}}}^{\mathcal{X}^{r}}$. Let $\left\{F_{i}\right\}_{i=1}^{\infty}$ be a Cauchy sequence in $F_{\mathcal{M}_{\omega, u^{r}}^{\mathcal{X}^{r}}}^{\mathcal{X}^{q}}$. Theorem 5.4 shows that $\left\{\mathrm{S}_{\varphi}\left(F_{i}\right)\right\}_{i=1}^{\infty}$ is a Cauchy sequence in $f_{\mathcal{M}_{\omega, u^{r}}^{\mathcal{X}^{r}}}^{\mathcal{X}^{q}}$. 
Therefore, $\lim _{i \rightarrow \infty} \mathrm{S}_{\varphi}\left(F_{i}\right)=s=\left\{s_{Q}\right\}_{Q \in \mathcal{Q}}$ exists. Define $F=\mathrm{T}_{\psi}(s)$. Theorem 5.4 and the identity $\mathrm{T}_{\psi} \circ \mathrm{S}_{\varphi}=$ id ensure that

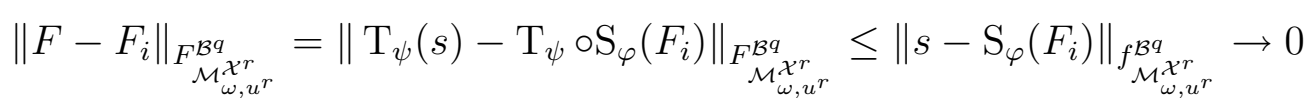

as $i$ goes to infinity. Hence, $F_{\mathcal{M}_{\omega, u^{r}}^{\mathcal{B}^{q}}}^{\mathcal{X}^{r}}$ is a quasi-Banach space.

We state and sketch the proof of the atomic decompositions of $F_{\mathcal{M}_{\omega, u^{r}}^{\mathcal{X}^{r}}}^{\mathcal{F}^{q}}$. The reader is referred to [24, (4.1)-(4.3) and (12.6)-(12.7)] for the definition of inhomogeneous smooth $N$-atom, $N \in \mathbf{N}$.

Theorem 5.7. Let $1 \leq p, q, r<\infty, N \in \mathbf{N}$ and $(\omega, u, \mathcal{X}, \mathcal{B}, \mathcal{B}) \in \mathbf{V}_{p}$. If $(\mathcal{X}, \mathcal{B})$ is admissible, then there exist a sequence $s=\left\{s_{Q}\right\}_{Q \in \mathcal{Q}} \in f_{\mathcal{M}_{\omega, u^{r}}^{\mathcal{X}^{r}}}^{\mathcal{B}^{q}}$ and a family of smooth $N$-atoms $\left\{a_{Q}\right\}_{Q \in \mathcal{Q}}$ so that $f=\sum_{Q \in \mathcal{Q}} s_{Q} a_{Q}$ and $\|s\|_{f_{\mathcal{M}_{\omega, u^{r}}^{\mathcal{B} q}}^{\mathcal{X}^{r}}} \leq C\|f\|_{F_{\mathcal{M}_{\omega, u^{r}}^{\mathcal{B} q}}^{\mathcal{X}^{r}}}$ for some constant $C>0$.

Proof. Using the ideas from [25, Theorem 5.11 and Lemma 5.12], for any $f \in$ $\mathcal{S}^{\prime}\left(\mathbf{R}^{n}\right)$, we have $f=\sum_{Q \in \mathcal{Q}} s_{Q} a_{Q}$ where $\left\{a_{Q}\right\}_{Q \in \mathcal{Q}}$ is a family of inhomogeneous smooth $N$-atoms and

$$
s_{Q}=|Q|^{1 / 2} \sup _{y \in Q}\left|\left(\varphi_{j} * f\right)(y)\right|
$$

where $\Phi=\left(\varphi_{0}, \varphi\right)$ satisfies the conditions in Definition 5.2. Furthermore, using the idea from Peetre's inequality again, when $m$ is large enough, we find that

$$
\sum_{|Q|=2^{-j n}} s_{Q} \tilde{\chi}_{Q}=\sum_{|Q|=2^{-j n}} \sup _{y \in Q}\left|\left(\varphi_{j} * f\right)(y)\right| \chi_{Q} \leq C\left(\eta_{j, m} *\left|\varphi_{j} * f\right|^{h}\right)^{\frac{1}{h}}
$$

where $h$ is given by the proof of Theorem 5.4. The admissibility of $(\mathcal{X}, \mathcal{B})$ and

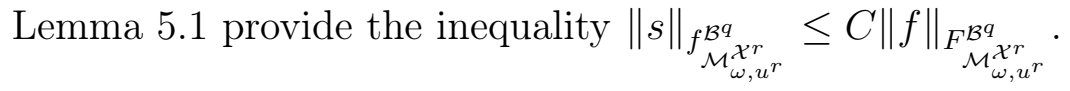

Definition 5.7. Let $0<\theta, \beta<\infty, 1 \leq p, q, r<\infty,(\omega, u, \mathcal{X}, \mathcal{B}, \mathcal{B}) \in \mathbf{V}_{p}$ and $(\mathcal{X}, \mathcal{B})$ be admissible with order $(\theta, \beta)$. We call that $\left\{m_{Q}\right\}_{Q \in \mathcal{Q}}$ is a family of smooth molecules for $F_{\mathcal{M}_{\omega, u^{r}}^{\mathcal{X}^{r}}}^{\mathcal{R}^{r}}$ if there exist $\delta>0$ and $M_{0}>J$ such that

$$
\begin{aligned}
\int x^{\lambda} m_{Q}(x) d x= & 0, \quad|\lambda| \leq N, \quad|Q|<1, \\
\left|\partial^{\gamma} m_{Q}(x)\right| \leq & |Q|^{-1 / 2-|\gamma| / n}\left(1+l(Q)^{-1}\left|x-x_{Q}\right|\right)^{-M_{0}}, \quad|\gamma| \leq[q \theta], \\
\left|\partial^{\gamma} m_{Q}(x)-\partial^{\gamma} m_{Q}(y)\right| \leq & |Q|^{-1 / 2-|\gamma| / n-\delta / n}|x-y|^{\delta} \\
& \times \sup _{|z| \leq|x-y|}\left(1+l(Q)^{-1}\left|x-z-x_{Q}\right|\right)^{-M_{0}}, \quad|\gamma|=[q \theta],
\end{aligned}
$$

where $J=\frac{\max (M, n+\Gamma)}{s_{0}} \max (q, r, 1)$ and $N=[J-n-q \beta]$.

For instance, when $\mathcal{X}=L^{p}\left(\mathbf{R}^{n}\right)$ and $\mathcal{B}(x)=l^{\alpha, s}$, we have $J=\frac{n}{\min (1, p, s)}$ and $N=[J-n-\alpha]$ which are precisely the results given in [24].

Theorem 5.8. Let $0<\theta, \beta<\infty, 1 \leq p, q, r<\infty$ and $(\omega, u, \mathcal{X}, \mathcal{B}, \mathcal{B}) \in \mathbf{V}_{p}$. Suppose that $(\mathcal{X}, \mathcal{B})$ is admissible with order $(\theta, \beta)$. If $f=\sum_{Q \in \mathcal{Q}} s_{Q} m_{Q}$ converges 
in $\mathcal{S}^{\prime}\left(\mathbf{R}^{n}\right)$ where $\left\{m_{Q}\right\}_{Q \in \mathcal{Q}}$ is a family of smooth molecules for $F_{\mathcal{M}_{\omega, u^{r}}^{\mathcal{X}^{q}}}^{\mathcal{X}^{r}}$, then

$$
\|f\|_{F_{\mathcal{M}_{\omega, u^{r}}^{\mathcal{B} q}}^{\mathcal{X}^{r}}} \leq C\|s\|_{f_{\mathcal{M}_{\omega, u^{r}}^{\mathcal{B} q}}^{\mathcal{X}^{r}}}
$$

for some constant $C>0$.

Proof. Let $\left(\varphi_{0}, \varphi\right)$ satisfy the conditions in Definition 5.2 and $Q \in \mathcal{Q}$ with $|Q|=2^{-\mu n}$. We find that $\left|\varphi_{\nu} * f(x)\right| \leq I_{\nu}+I I_{\nu}$ where

$$
\begin{aligned}
I_{\nu} & =\sum_{\mu=0}^{\nu-1} \sum_{l(Q)=2^{-\mu}}\left|s_{Q}\right|\left|m_{Q} * \varphi_{\nu}(x)\right|, \\
I I_{\nu} & =\sum_{\mu=\nu}^{\infty} \sum_{l(Q)=2^{-\mu}}\left|s_{Q}\right|\left|m_{Q} * \varphi_{\nu}(x)\right| .
\end{aligned}
$$

When $\nu \leq \mu$, according to [24, Lemmas 3.6 and B.1], we assert that

$$
\left|m_{Q} * \varphi_{\nu}(x)\right| \leq C 2^{\frac{n \nu}{2}} 2^{(\nu-\mu)\left(J+\frac{\epsilon}{2}-q \beta-\frac{n}{2}\right)}\left(1+2^{\nu}\left|x_{Q}-x\right|\right)^{-J-\epsilon}
$$

for some $\epsilon>0$.

For $I I_{\nu}$, we obtain

$$
\begin{aligned}
I I_{\nu} & \leq C 2^{\frac{n \nu}{2}} \sum_{\mu=\nu}^{\infty} 2^{(\nu-\mu)\left(J+\frac{\epsilon}{2}-q \beta-\frac{n}{2}\right)} \sum_{l(Q)=2^{-\mu}}\left|s_{Q}\right| /\left(1+2^{\nu}\left|x-x_{Q}\right|\right)^{J+\epsilon} \\
& \leq C \sum_{\mu=\nu}^{\infty} 2^{(\mu-\nu)\left(q \beta-\frac{\epsilon}{2}\right)}\left(\left(\sum_{k \in \mathbf{Z}^{n}}\left|s_{Q_{\mu, k}}\right| \tilde{\chi}_{Q_{\mu, k}}\right)^{h} * \eta_{\mu, h J+h \epsilon}\right)^{\frac{1}{h}}
\end{aligned}
$$

where $h$ is slightly less than $\min \left(\frac{s_{0}}{r}, \frac{s_{0}}{q}\right)$ so that $h J+h \epsilon>\max (M, n+\Gamma)$. We have the last inequality because

$$
2^{\mu}|x-y| \leq 2^{\mu-\nu}\left(2^{\nu}\left|x-x_{Q}\right|+2\right), \quad \forall y \in Q .
$$

Similarly, when $\mu \leq \nu$, we have

$$
\left|m_{Q} * \varphi_{\nu}(x)\right| \leq C 2^{\frac{n \nu}{2}} 2^{(\mu-\nu)\left(q \theta+\frac{n}{2}+\frac{\epsilon}{2}\right)}\left(1+2^{\mu}\left|x_{Q}-x\right|\right)^{-J-\epsilon} .
$$

Thus,

$$
I_{\nu} \leq C \sum_{\mu=0}^{\nu-1} 2^{(\mu-\nu)\left(q \theta+\frac{\epsilon}{2}\right)}\left(\left(\sum_{k \in \mathbf{Z}^{n}}\left|s_{Q_{\mu, k}}\right| \tilde{\chi}_{Q_{\mu, k}}\right)^{h} * \eta_{\mu, h J+h \epsilon}\right)^{\frac{1}{h}} .
$$

The boundedness of $\vec{S}_{\theta}$ and $\overleftarrow{S}_{\beta}$ on $\mathcal{B}$ offer the boundedness of $\vec{S}_{q \theta}$ and $\overleftarrow{S}_{q \beta}$ on $\mathcal{B}^{q}$ when $q \geq 1$. Thus, the admissibility condition yields the convergence of the molecular expansion and inequality (5.7).

\section{Function spaces of variable smoothness and integrability}

One of the pioneer studies of the variable exponent analysis is [44]. The main family of function spaces considered in [44] is the variable Lebesgue spaces. 
Definition 6.1. Let $p(x): \mathbf{R}^{n} \rightarrow(0, \infty)$ be a Lebesgue measurable function. The variable Lebesgue space $L^{p(\cdot)}\left(\mathbf{R}^{n}\right)$ consists of all Lebesgue measurable functions $f: \mathbf{R}^{n} \rightarrow \mathbf{C}$ so that

$$
\|f\|_{L^{p(\cdot)}\left(\mathbf{R}^{n}\right)}=\inf \left\{\lambda>0: \int_{\mathbf{R}^{n}}\left|\frac{f(x)}{\lambda}\right|^{p(x)} d x \leq 1\right\}<\infty .
$$

We call $p(x)$ the exponent function of $L^{p(\cdot)}\left(\mathbf{R}^{n}\right)$.

The variable Lebesgue space is a Banach space when $1 \leq p(x) \leq \infty$ (see [44, Theorem 2.5]). The following is a fundamental result for variable Lebesgue spaces given in [44, Theorem 2.6].

Theorem 6.1. If $1<p(x)<\infty$, then the associate space of $L^{p(\cdot)}\left(\mathbf{R}^{n}\right)$ is $L^{p^{\prime}(\cdot)}\left(\mathbf{R}^{n}\right)$ where $p^{\prime}$ satisfies $\frac{1}{p(x)}+\frac{1}{p^{\prime}(x)}=1$.

We call $p^{\prime}(x)$ the conjugate function of $p(x)$.

The monotone convergence theorem shows that $L^{p(\cdot)}\left(\mathbf{R}^{n}\right)$ fulfills condition (3) of Definition 2.1. As $\chi_{E} \in L^{p(\cdot)}\left(\mathbf{R}^{n}\right) \cap L^{p^{\prime}(\cdot)}\left(\mathbf{R}^{n}\right)$ when $|E|<\infty, L^{p(\cdot)}\left(\mathbf{R}^{n}\right)$ is a B.f.s.

One of the important breakthroughs on the variable exponent analysis is the establishment of the boundedness of the maximal operator on variable Lebesgue spaces given in $[15,17,57]$. The following gives the condition on the exponent functions of the variable Lebesgue spaces $L^{p(\cdot)}\left(\mathbf{R}^{n}\right)$ so that the maximal operator is bounded on $L^{p(\cdot)}\left(\mathbf{R}^{n}\right)$.

Definition 6.2. A continuous function $g$ on $\mathbf{R}^{n}$ is locally log-Hölder continuous if there exists $c_{l o g}>0$ such that

$$
|g(x)-g(y)| \leq \frac{c_{\log }}{\log (e+1 /|x-y|)}, \quad \forall x, y \in \mathbf{R}^{n} .
$$

We denote the class of locally log-Hölder continuous function by $C_{l o c}^{\log }\left(\mathbf{R}^{n}\right)$.

Furthermore, a continuous function is globally log-Hölder continuous if $g \in$ $C_{l o c}^{\log }\left(\mathbf{R}^{n}\right)$ and there exists $g_{\infty} \in \mathbf{R}$ so that

$$
\left|g(x)-g_{\infty}\right| \leq \frac{c_{\log }}{\log (e+1 /|x-y|)}, \quad \forall x \in \mathbf{R}^{n} .
$$

The class of globally log-Hölder continuous function is denoted by $C^{\log }\left(\mathbf{R}^{n}\right)$.

For any Lebesgue measurable function $p(x): \mathbf{R}^{n} \rightarrow(0, \infty)$, define $p_{-}=\inf _{x \in \mathbf{R}^{n}}$ $p(x)$ and $p_{+}=\sup _{x \in \mathbf{R}^{n}} p(x)$.

The succeeding result is now a well-known fact. For the proof of the following theorem, the reader is referred to $[15,17,57]$.

Theorem 6.2. If $p \in C^{\log }\left(\mathbf{R}^{n}\right)$ and $1<p_{-} \leq p_{+}<\infty$, then the HardyLittlewood maximal operator

$$
(\mathrm{M} f)(x)=\sup _{r>0} \frac{1}{|B(x, r)|} \int_{B(x, r)}|f(y)| d y
$$

is bounded from $L^{p(\cdot)}\left(\mathbf{R}^{n}\right)$ to $L^{p(\cdot)}\left(\mathbf{R}^{n}\right)$.

The above result provides an access for us to apply the results from the previous sections to $L^{p(\cdot)}\left(\mathbf{R}^{n}\right)$. We first show that $L^{p(\cdot)}\left(\mathbf{R}^{n}\right)$ is a tempered B.f.s. whenever $p \in C^{\log }\left(\mathbf{R}^{n}\right)$ and $1<p_{-} \leq p_{+}<\infty$. 
Lemma 6.3. If $p \in C^{\log }\left(\mathbf{R}^{n}\right)$ and $1<p_{-} \leq p_{+}<\infty$, then $L^{p(\cdot)}\left(\mathbf{R}^{n}\right)$ is a tempered B.f.s. with $e_{L^{p(\cdot)}\left(\mathbf{R}^{n}\right)}=p_{+}, e_{L^{p(\cdot)}\left(\mathbf{R}^{n}\right)}^{\prime}=p_{-}^{\prime}$ and $\left(|\cdot|, u, L^{p(\cdot)}\left(\mathbf{R}^{n}\right)\right) \in \mathbf{M}_{1}$ provided that $u \in \mathcal{W}_{p_{+}}$.

Proof. As $p \in C^{\log }\left(\mathbf{R}^{n}\right)$ and $1<p_{-} \leq p_{+}<\infty$, we find that

$$
p \in C^{\log }\left(\mathbf{R}^{n}\right) \Longrightarrow p^{\prime} \in C^{\log }\left(\mathbf{R}^{n}\right)
$$

and $1<p_{-}^{\prime} \leq p_{+}^{\prime}<\infty$.

We have $\left(L^{p(\cdot)}\left(\mathbf{R}^{n}\right)^{\prime}\right)^{q}=L^{p^{\prime}(\cdot) / q}\left(\mathbf{R}^{n}\right)$ and $p^{\prime}(\cdot) / q \in C^{\log }\left(\mathbf{R}^{n}\right)$ for any $0<q<$ $\infty$. Thus, $L^{p(\cdot)}\left(\mathbf{R}^{n}\right)$ is a tempered B.f.s. with $e_{L^{p(\cdot)}\left(\mathbf{R}^{n}\right)}^{\prime}=p_{-}^{\prime}$. For the exponent of $L^{p(\cdot)}\left(\mathbf{R}^{n}\right)$, we assert that

$$
e_{L^{p(\cdot)}\left(\mathbf{R}^{n}\right)}=\frac{e_{L^{p(\cdot)}\left(\mathbf{R}^{n}\right)}^{\prime}}{e_{L^{p(\cdot)}\left(\mathbf{R}^{n}\right)}^{\prime}-1}=\frac{p_{-}^{\prime}}{p_{-}^{\prime}-1}=p_{+} .
$$

The final assertion follows from the fact that the Lebesgue measure belongs to $A_{1}$ and $L^{p(\cdot)}\left(\mathbf{R}^{n}\right)$ is 1-convex.

We provide two remarkable properties for the variable Lebesgue spaces based on the results from Section 5 .

Definition 6.3. Let $p \in L^{\infty}$. For any $B \in \mathbf{B}$, define $\bar{p}_{B}$ by

$$
\frac{1}{\bar{p}_{B}}=\frac{1}{|B|} \int_{B} \frac{1}{p(x)} d x \text {. }
$$

Proposition 6.4. Let $p \in C^{\log }\left(\mathbf{R}^{n}\right)$ and $1<p_{-} \leq p_{+}<\infty$. There exist $C_{1}, C_{2}>0$ so that for any $B \in \mathbf{B}$,

$$
C_{1}|B|^{\frac{1}{\bar{p}_{B}}} \leq\left\|\chi_{B}\right\|_{L^{p(\cdot)}\left(\mathbf{R}^{n}\right)} \leq C_{2}|B|^{\frac{1}{\bar{p}_{B}}} .
$$

Hence, $L^{p(\cdot)}\left(\mathbf{R}^{n}\right)$ is of polynomial growth with $\beta=1 / p_{-}$.

Proof. By [18, Lemma 3.4], we have a constant $C_{2}$ so that $\left\|\chi_{B}\right\|_{L^{p(\cdot)}\left(\mathbf{R}^{n}\right)} \leq$ $C_{2}|B|^{\frac{1}{\bar{p}_{B}}}$. Furthermore, as $L^{p(\cdot)}\left(\mathbf{R}^{n}\right)$ is a tempered B.f.s., Proposition 2.4 and the fact that

$$
\frac{1}{\bar{p}_{B}}+\frac{1}{\overline{p^{\prime}}}=\frac{1}{|B|}\left(\int_{B} \frac{1}{p(x)}+\frac{1}{p^{\prime}(x)} d x\right)=1
$$

guarantee the validity of the first inequality in (6.1).

Similarly, applying Proposition 2.5 to $L^{p(\cdot)}\left(\mathbf{R}^{n}\right)$ with $p \in C^{\log }\left(\mathbf{R}^{n}\right)$ and $1<p_{-} \leq$ $p_{+}<\infty$, we obtain the subsequent results.

Proposition 6.5. Let $p \in C^{\log }\left(\mathbf{R}^{n}\right)$ and $1<p_{-} \leq p_{+}<\infty$. For any $1 \leq q<p_{-}^{\prime}$ and $1 \leq p<p_{-}$, there exist constants $C_{1}, C_{2}>0$ such that for any $x_{0} \in \mathbf{R}^{n}$ and $r>0$, we have

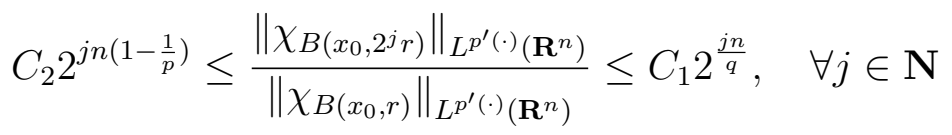

and

$$
C_{2} 2^{j n\left(1-\frac{1}{q}\right)} \leq \frac{\left\|\chi_{B\left(x_{0}, 2^{j} r\right)}\right\|_{L^{p(\cdot)}\left(\mathbf{R}^{n}\right)}}{\left\|\chi_{B\left(x_{0}, r\right)}\right\|_{L^{p(\cdot)}\left(\mathbf{R}^{n}\right)}} \leq C_{1} 2^{\frac{j n}{p}}, \quad \forall j \in \mathbf{N}
$$


Some similar inequalities are obtained in [37, Lemma 1]. We now give the definition of variable Morrey spaces.

Definition 6.4. Let $p(x): \mathbf{R}^{n} \rightarrow(0, \infty)$ be a Lebesgue measurable function and $u \in \mathcal{W}_{p_{+}}$. The variable Morrey space $\mathcal{M}_{u}^{p(\cdot)}$ consists of all Lebesgue measurable functions $f: \mathbf{R}^{n} \rightarrow \mathbf{C}$ so that

$$
\|f\|_{\mathcal{M}_{u}^{p(\cdot)}}=\sup _{z \in \mathbf{R}^{n}, R>0} \frac{1}{u(z, R)}\left\|\chi_{B(z, R)} f\right\|_{L^{p(\cdot)}\left(\mathbf{R}^{n}\right)}<\infty .
$$

The above definition includes the variable Morrey spaces introduced in [41] as a special case if the bounded open set used in Definition 3.1 of [41] is replaced by $\mathbf{R}^{n}$.

We have the subsequent generalization of [14, Corollary 2.5].

Corollary 6.6. Let $p \in C^{\log }\left(\mathbf{R}^{n}\right)$ and $u \in \mathcal{W}_{p_{+}}$. Suppose that $T(f)=K * f$ where $K$ is a locally integrable function defined on $\mathbf{R}^{n} \backslash\{0\}$ with $|\hat{K}(\xi)| \leq C$,

$$
|K(x)| \leq \frac{C}{|x|^{n}} \quad \text { and } \quad|\nabla K(x)| \leq \frac{C}{|x|^{n+1}}, \quad x \neq 0
$$

for some $C>0$. Then, for any $1<q<\infty$, there exists a constant $C>0$ such that

$$
\left\|\left(\sum_{j \in \mathbf{N}}\left|T f_{j}\right|^{q}\right)^{\frac{1}{q}}\right\|_{\mathcal{M}_{u}^{p(\cdot)}} \leq C\left\|\left(\sum_{j \in \mathbf{N}}\left|f_{j}\right|^{q}\right)^{\frac{1}{q}}\right\|_{\mathcal{M}_{u}^{p(\cdot)}} .
$$

Since $\left(|\cdot|, u, L^{p(\cdot)}\left(\mathbf{R}^{n}\right)\right) \in \mathbf{M}_{1}$ when $u \in \mathcal{W}_{p_{+}}$, the preceding corollary is proved by applying the lifting principle to the singular integral operators in [14, Corollary 2.5]. In fact, the weighted version of the above result is also valid if we apply the lifting principle to the corresponding results in [42]. For brevity, we leave the details to the reader.

The following theorem is a consequence of Corollary 4.3. It gives an extension of the result in [20, Theorem 4.2] to $\mathcal{M}_{u}^{p(\cdot)}$.

Theorem 6.7. Let $p \in C^{\log }\left(\mathbf{R}^{n}\right)$ and $u \in \mathcal{W}_{p_{+}}$. If $1<p_{-} \leq p_{+}<\infty$, then $\mathcal{M}_{u}^{p(\cdot)}$ possesses the Littlewood-Paley characterization.

Proof. According to Proposition 6.4, $L^{p(\cdot)}\left(\mathbf{R}^{n}\right)$ is a B.f.s. of polynomial growth. Lemma 4.1 assures that $\mathcal{S}_{0}\left(\mathbf{R}^{n}\right) \hookrightarrow \mathcal{M}_{u}^{p(\cdot)} \hookrightarrow \mathcal{S}^{\prime}\left(\mathbf{R}^{n}\right) / \mathcal{P}$. In addition, [20, Theorem 4.2] ensures that both $L^{p(\cdot)}\left(\mathbf{R}^{n}\right)$ and $L^{p^{\prime}(\cdot)}\left(\mathbf{R}^{n}\right)$ possess the Littlewood-Paley characterization. That is, the operator $\mathcal{G}_{\varphi}$ is bounded from $L^{p(\cdot)}\left(\mathbf{R}^{n}\right)$ to $L^{p(\cdot)}\left(\mathbf{R}^{n}\right)\left(l^{2}\right)$ and from $L^{p^{\prime}(\cdot)}\left(\mathbf{R}^{n}\right)$ to $L^{p^{\prime}(\cdot)}\left(\mathbf{R}^{n}\right)\left(l^{2}\right)$. Thus, Corollary 4.3 asserts that $\mathcal{M}_{u}^{p(\cdot)}$ possesses the Littlewood-Paley characterization.

We adopt the Standing Assumption introduced in [20] for the study of variable Morrey spaces.

Definition 6.5. The Lebesgue measurable functions $p(x), q(x), \alpha(x): \mathbf{R}^{n} \rightarrow$ $(0, \infty)$ satisfy the Standing Assumptions if $p, q \in C^{\log }\left(\mathbf{R}^{n}\right)$ with $0<p_{-} \leq p_{+}<\infty$ and $0<q_{-} \leq q_{+}<\infty, \alpha \in C_{\operatorname{loc}}^{\log }\left(\mathbf{R}^{n}\right) \cap L^{\infty}$ and that $\lim _{x \rightarrow \infty} \alpha(x)$ exists. We write $(p, q, \alpha) \in \mathbf{S}$ if they satisfy the Standing Assumptions. 
Let $q(x) \in C^{\log }\left(\mathbf{R}^{n}\right)$ with $0<q_{-} \leq q_{+}<\infty$. For any family of Lebesgue measurable functions $\left\{f_{\nu}\right\}_{\nu \in \mathbf{N}}$, define

$$
\left\|f_{\nu}(x)\right\|_{l_{\nu}^{q(x)}}=\left(\sum_{\nu=0}^{\infty}\left|f_{\nu}(x)\right|^{q(x)}\right)^{\frac{1}{q(x)}}
$$

and $l_{\nu}^{q(\cdot)}=\left\{l_{\nu}^{q(x)}\right\}_{x \in \mathbf{R}^{n}}$. Moreover, let $\alpha$ be as in the Standing Assumptions, we define

$$
\left\|f_{\nu}(x)\right\|_{l_{\nu}^{\alpha(x), q(x)}}=\left(\sum_{\nu=0}^{\infty}\left(2^{\nu \alpha(x)}\left|f_{\nu}(x)\right|\right)^{q(x)}\right)^{\frac{1}{q(x)}}
$$

and $l_{\nu}^{\alpha(\cdot), q(\cdot)}=\left\{l_{\nu}^{\alpha(x), q(x)}\right\}_{x \in \mathbf{R}^{n}}$. Whenever $1<q_{-} \leq q_{+}<\infty$ and $\alpha$ satisfies the Standing Assumptions, $l_{\nu}^{\alpha(\cdot), q(\cdot)}$ is a family of variable Banach sequence spaces.

Lemma 6.8. Let $p \in C^{\log }\left(\mathbf{R}^{n}\right)$ with $1<p_{-} \leq p_{+}<\infty$ and $u \in \mathcal{W}_{p_{+}}$. If $(\alpha, p, q) \in \mathbf{S}$ and $1<q_{-} \leq q_{+}<\infty$, then $\left(|\cdot|, u, L^{p(\cdot)}\left(\mathbf{R}^{n}\right), l_{\nu}^{\alpha(\cdot), q(\cdot)}, l_{\nu}^{\alpha(\cdot), q(\cdot)}\right) \in \mathbf{V}_{1}$.

Proof. When $q(y) \leq q(x)$ and $\alpha(x) \leq \alpha(y)$, we have the embedding $l_{\nu}^{\alpha(y), q(y)} \hookrightarrow$ $l_{\nu}^{\alpha(x), q(x)}$. Therefore, (5.1) is fulfilled with $\gamma(x, y)=0$.

If $q(x)<q(y)$, then Hölder's inequality guarantees that

$$
\left\|\left\{2^{-\frac{\nu}{r(x, y)}} a_{\nu}\right\}_{\nu=0}^{\infty}\right\|_{l_{\nu}^{q(x)}} \leq\left\|\left\{2^{-\frac{\nu}{r(x, y)}}\right\}_{\nu=0}^{\infty}\right\|_{l_{\nu}^{r(x, y)}}\left\|\left\{a_{\nu}\right\}_{\nu=0}^{\infty}\right\|_{l_{\nu}^{q(y)}}
$$

where $\frac{1}{q(x)}=\frac{1}{r(x, y)}+\frac{1}{q(y)}$. Moreover, $|x-y|^{\frac{1}{q(y)}-\frac{1}{q(x)}}$ is bounded above because $q \in$ $C^{\log }\left(\mathbf{R}^{n}\right)$ with $1<q_{-} \leq q_{+}<\infty$ implies $\frac{1}{q} \in C^{\log }\left(\mathbf{R}^{n}\right)$. As $0<\frac{1}{r(x, y)} \leq \frac{1}{q_{-}}-\frac{1}{q_{+}}$, we have inequality (5.1) for $l_{\nu}^{q(x)}$ with $\gamma(x, y)=\frac{1}{r(x, y)}=\frac{1}{q(x)}-\frac{1}{q(y)}$.

Similarly, when $\alpha(y)<\alpha(x)$, we find that $|x-y|^{\alpha(y)-\alpha(x)}$ is bounded above in view of $\alpha \in C_{l o c}^{\log }\left(\mathbf{R}^{n}\right) \cap L^{\infty}\left(\mathbf{R}^{n}\right)$.

In conclusion, (5.1) is valid for $l_{\nu}^{\alpha(\cdot), q(\cdot)}$ with

$$
\gamma(x, y)=(\alpha(x)-\alpha(y)) \chi_{\left\{(x, y) \in \mathbf{R}^{2 n}: \alpha(y)<\alpha(x)\right\}}+\left(\frac{1}{q(x)}-\frac{1}{q(y)}\right) \chi_{\left\{(x, y) \in \mathbf{R}^{2 n}: q(x)<q(y)\right\}} .
$$

The previous lemma shows that the function space $C^{\log }\left(\mathbf{R}^{n}\right)$ is not only related to the boundedness of the maximal operator on $L^{p(\cdot)}\left(\mathbf{R}^{n}\right)$ but also offers a condition for the study of vector-valued singular integral operators on $L^{p(\cdot)}\left(\mathbf{R}^{n}\right)$ as demonstrated in the proof of Lemma 5.1.

We give the definitions of variable Triebel-Lizorkin-Morrey spaces and the corresponding sequence spaces.

Definition 6.6. Let $(p, q, \alpha) \in \mathbf{S}$ and $u \in \mathcal{W}_{p_{+}}$. The variable Triebel-LizorkinMorrey space $\mathcal{E}_{p(\cdot), q(\cdot), u}^{\alpha \cdot)}$ consists of those $f \in \mathcal{S}^{\prime}\left(\mathbf{R}^{n}\right)$ satisfying

$$
\|f\|_{\mathcal{E}_{p(\cdot), q(\cdot), u}^{\alpha(\cdot)}}=\|\|\left\{2^{\nu \alpha(x)}\left(f * \varphi_{\nu}\right)(x)\right\}_{\nu=0}^{\infty}\left\|_{l_{\nu}^{q(x)}}\right\|_{\mathcal{M}_{u}^{p(\cdot)}}<\infty
$$

where the pair $\Phi=\left(\varphi_{0}, \varphi\right)$ satisfies the conditions in Definition 5.2.

When $u \equiv 1$, the variable Triebel-Lizorkin-Morrey spaces reduce to the TriebelLizorkin spaces $F_{p(\cdot), q(\cdot)}^{\alpha(\cdot)}$ defined in [20]. In addition, the "inhomogeneous" version of the variable Triebel-Lizorkin spaces introduced in [75] is a member of $\mathcal{E}_{p(\cdot), q(\cdot), u}^{\alpha(\cdot)}$. 
On one hand, the family of variable Triebel-Lizorkin-Morrey spaces is an extension of the Triebel-Lizorkin-Morrey spaces in [52, 66, 71, 74]. On the other hand, it generalizes the function spaces in $[20,75]$ by replacing the underlying variable Lebesgue spaces by variable Morrey spaces.

As shown in Section 5, we need an analogue family of sequence spaces for the variable Triebel-Lizorkin-Morrey spaces.

Definition 6.7. Let $(p, q, \alpha) \in \mathbf{S}$ and $u \in \mathcal{W}_{p_{+}}$. The variable Triebel-LizorkinMorrey sequence space $e_{p(\cdot), q(\cdot), u}^{\alpha(\cdot)}$ consists of those complex-valued sequence $s=\left\{s_{Q}\right\}_{Q \in \mathcal{Q}}$ satisfying

$$
\|s\|_{e_{p(\cdot), q(\cdot), u}^{\alpha(\cdot)}}=\|\| 2^{\nu \alpha(x)}\left\{\sum_{k \in \mathbf{Z}^{n}}\left|s_{Q_{\nu, k}}\right| \tilde{\chi}_{Q_{\nu, k}}\right\}_{\nu=0}^{\infty}\|\|_{l_{\nu}^{q(x)}} \|_{\mathcal{M}_{u}^{p(\cdot)}}<\infty .
$$

In order to apply the results form Section 5 to the variable Triebel-LizorkinMorrey spaces, we verify the admissibility of $\left(L^{p(\cdot)}\left(\mathbf{R}^{n}\right), l_{\nu}^{\alpha(\cdot), q(\cdot)}\right)$ in the following lemma.

Lemma 6.9. Let $u \in \mathcal{W}_{p_{+}}$and $(p, q, \alpha) \in \mathbf{S}$ with $1<p_{-} \leq p_{+}<\infty$ and $1<q_{-} \leq q_{+}<\infty$. Then $\left(L^{p(\cdot)}\left(\mathbf{R}^{n}\right), l_{\nu}^{\alpha(\cdot), q(\cdot)}\right)$ is admissible with order $\left(\alpha_{+}, \alpha_{-}\right)$.

Proof. According to [20, Theorem 3.2], we have

$$
\left\|\left\{\eta_{j, m} * f_{j}\right\}_{j=0}^{\infty}\right\|_{L^{p(\cdot)}\left(\mathbf{R}^{n}\right)\left(l_{\nu}^{q(\cdot)}\right)} \leq B\left\|\left\{f_{j}\right\}_{j=0}^{\infty}\right\|_{L^{p(\cdot)}\left(\mathbf{R}^{n}\right)\left(l_{\nu}^{q(\cdot)}\right)}
$$

whenever $m>n$. Furthermore,

$$
\begin{aligned}
\left\|\left\{\eta_{j, 2 m} * f_{j}\right\}_{j=0}^{\infty}\right\|_{L^{p(\cdot)}\left(\mathbf{R}^{n}\right)\left(l_{\nu}^{q(\cdot), \alpha(\cdot)}\right)} & =\left\|\left\{2^{j \alpha(x)}\left(\eta_{j, 2 m} * f_{j}\right)\right\}_{j=0}^{\infty}\right\|_{L^{p(\cdot)}\left(\mathbf{R}^{n}\right)\left(l_{\nu}^{q(x)}\right)} \\
& \left.\leq C \| \eta_{j, m} *\left(2^{j \alpha(\cdot)}\left|f_{j}\right|\right)\right\}_{j=0}^{\infty} \|_{L^{p(\cdot)}\left(\mathbf{R}^{n}\right)\left(l_{\nu}^{q(\cdot)}\right)} .
\end{aligned}
$$

We have the last inequality because [20, Lemma 6.1] gives

$$
2^{\nu \alpha(x)} \eta_{\nu, 2 m}(x-y) \leq C 2^{\nu \alpha(y)} \eta_{\nu, m}(x-y), \quad x, y \in \mathbf{R}^{n} .
$$

Consequently, inequality (6.2) guarantees that

$$
\left\|\left\{\eta_{j, 2 m} * f_{j}\right\}_{j=0}^{\infty}\right\|_{L^{p(\cdot)}\left(\mathbf{R}^{n}\right)\left(l_{\nu}^{q(\cdot), \alpha(\cdot)}\right)} \leq C\left\|\left\{f_{j}\right\}_{j=0}^{\infty}\right\|_{L^{p(\cdot)}\left(\mathbf{R}^{n}\right)\left(l_{\nu}^{q(\cdot), \alpha(\cdot)}\right)} .
$$

We find that $\left(L^{p(\cdot)}\left(\mathbf{R}^{n}\right)\right)^{s}=L^{p(\cdot) / s}\left(\mathbf{R}^{n}\right)$ and $\left(l_{\nu}^{q(\cdot), \alpha(\cdot)}\right)^{t}=l_{\nu}^{q(\cdot) / t, t \alpha(\cdot)}$ for any $0<s, t<$ $\infty$. Thus, $\left(L^{p(\cdot)}\left(\mathbf{R}^{n}\right), l_{\nu}^{\alpha(\cdot), q(\cdot)}\right)$ satisfies Condition (1) of Definition 5.6.

Finally, we show that $l_{\nu}^{\alpha(\cdot), q(\cdot)}$ fulfills Condition (2) of Definition 5.6. For any $a=\left\{a_{j}\right\}_{j=0}^{\infty}$, we have

$$
2^{i \alpha(x)} \sum_{j=0}^{i} 2^{(j-i)\left(\alpha_{+}+\epsilon\right)} a_{j}=\sum_{j=0}^{i} 2^{(j-i)\left(\alpha_{+}+\epsilon-\alpha(x)\right)} 2^{j \alpha(x)} a_{j} .
$$

Young's inequality assures that

$$
\begin{aligned}
\left\|\vec{S}_{\alpha_{+}}(a)\right\|_{l_{i}^{\alpha(x), q(x)}} & =\left\|\left\{2^{i \alpha(x)} \sum_{j=0}^{i} 2^{(j-i)\left(\alpha_{+}+\epsilon\right)} a_{j}\right\}_{i=0}^{\infty}\right\|_{l_{i}^{q(x)}} \\
& \leq C\left\|\left\{2^{j \alpha(x)} a_{j}\right\}_{j=0}^{\infty}\right\|_{l_{j}^{q(x)}}=\|a\|_{l_{i}^{\alpha(x), q(x)}}
\end{aligned}
$$


for some $C>0$ independent of $x$ because $1<q_{-} \leq q_{+}<\infty$. Similarly, we obtain $\left\|\overleftarrow{S}_{\alpha_{-}}(a)\right\|_{l_{j}^{\alpha(x), q(x)}} \leq C\|a\|_{l_{j}^{\alpha(x), q(x)}}$ for some $C>0$ independent of $x$. Therefore, we conclude that $\left(L^{p(\cdot)}\left(\mathbf{R}^{n}\right), l_{\nu}^{\alpha(\cdot), q(\cdot)}\right)$ is admissible with order $\left(\alpha_{+}, \alpha_{-}\right)$.

Using the results from Section 5, we obtain the following theorems.

Theorem 6.10. Let $(p, q, \alpha) \in \mathbf{S}$ and $u \in \mathcal{W}_{p_{+}}$. The $\varphi$-transform $\mathrm{S}_{\varphi}: \mathcal{E}_{p(\cdot), q(\cdot), u}^{\alpha(\cdot)} \rightarrow$ $e_{p(\cdot), q(\cdot), u}^{\alpha(\cdot)}$ and the $\psi$-transform $\mathrm{T}_{\psi}: e_{p(\cdot), q(\cdot), u}^{\alpha(\cdot)} \rightarrow \mathcal{E}_{p(\cdot), q(\cdot), u}^{\alpha(\cdot)}$ are bounded. Moreover, $\mathcal{E}_{p(\cdot), q(\cdot), u}^{\alpha(\cdot)}$ and $e_{p(\cdot), q(\cdot), u}^{\alpha(\cdot)}$ are quasi-Banach spaces.

Theorem 6.11. Let $(p, q, \alpha) \in \mathbf{S}, N \in \mathbf{N}$ and $u \in \mathcal{W}_{p_{+}}$. There exist a sequence $s=\left\{s_{Q}\right\}_{Q \in \mathcal{Q}} \in e_{p(\cdot), q(\cdot), u}^{\alpha(\cdot)}$ and a family of smooth $N$-atoms $\left\{a_{Q}\right\}_{Q \in \mathcal{Q}}$ so that $f=$ $\sum_{Q \in \mathcal{Q}} s_{Q} a_{Q}$ and $\|s\|_{e_{p(\cdot), q(\cdot), u}^{\alpha(\cdot)}} \leq C\|f\|_{\mathcal{E}_{p(\cdot), q(\cdot), u}^{\alpha(\cdot)}}$ for some $C>0$.

Theorem 6.12. Let $f=\sum_{Q \in \mathcal{Q}} s_{Q} m_{Q}$ where $\left\{m_{Q}\right\}_{Q \in \mathcal{Q}}$ is a family of smooth molecules for $\mathcal{E}_{p(\cdot), q(\cdot), u}^{\alpha(\cdot)}$ Then

for some $C>0$.

$$
\|f\|_{\mathcal{E}_{p(\cdot), q(\cdot), u}^{\alpha(\cdot)}} \leq C\|s\|_{e_{p(\cdot), q(\cdot), u}^{\alpha(\cdot)}}
$$

As shown in [20, Theorem 3.8], the smoothness and the vanishing moment conditions imposed on the smooth molecules for $\mathcal{E}_{p(\cdot), q(\cdot), u}^{\alpha(\cdot)}$ when $u \equiv 1$ can be further refined. More precisely, the vanishing moment conditions and the smoothness conditions can be localized on each dyadic cube associated with the molecules. In fact, that refinement on the molecular decompositions for $\mathcal{E}_{p(\cdot), q(\cdot), u}^{\alpha(\cdot)}$ is also valid. On the other hand, the main focus of this paper is the applications of the lifting principle for singular integral operators on the weighted vector-valued Morrey spaces. The refinement relies heavily on some techniques specially adapted to the variable Lebesgue spaces and $l_{\nu}^{\alpha(\cdot), q(\cdot)}$. So, the improvement of the molecular decompositions for $\mathcal{E}_{p(\cdot), q(\cdot), u}^{\alpha(\cdot)}$ and the analysis of the convergence of the refined molecular expansions will appear elsewhere.

Acknowledgement. The author would like to thank the referee for careful reading of the paper and valuable comments.

\section{References}

[1] Acerbi, E., and G. Mingione: Regularity results for stationary electro-rheological fluids. Arch. Ration. Mech. Anal. 163, 2002, 213-259.

[2] Acerbi, E., and G. Mingione: Regularity results for electrorheological fluids: The stationary case. - C. R. Acad. Sci. Paris Ser. I 334, 2002, 817-822.

[3] Andersen, K., and R. John: Weighted inequalities for vector-valued maximal functions and singular integrals. - Studia Math. 69, 1980, 19-31.

[4] Almeida, A., J. Hasanov, and S. Samko: Maximal and potential operators in variable exponent Morrey spaces. - Georgian Math. J. 15, 2008, 195-208.

[5] Almeida, A., and P. HÄstö: Besov spaces with variable smoothness and integrability. - J. Funct. Anal. 258, 2010, 1628-1655.

[6] Benedek, A., A. Calderón, and R. Panzone: Convolution operators on Banach spaces valued functions. - Proc. Nat. Acad. Sci. U.S.A. 48, 1962, 356-365. 
[7] Bennett, C., and R. Sharpley: Interpolation of operators. - Academic Press, 1988.

[8] Blasco, O.: Some aspects of vector-valued singular integrals. - In: Recent Developments in Real and Harmonic Analysis, Birkhäuser, Boston, 2009, 37-56.

[9] Bourgain J.: Some remarks on Banach spaces in which martingale difference sequence are unconditional. - Ark. Mat. 21, 1983, 163-168.

[10] Bourgain J.: Extension of a result of Benedek, Calderón and Panzone. - Ark. Mat. 22, 1984, 91-95.

[11] Chen, Y., S. Levine, and R. Rao: Variable exponent, linear growth functionals in image restoration. - SIAM J. Appl. Math. 66, 2006, 1383-1406.

[12] Chinennza, F., and M. Frasca: Morrey spaces and Hardy-Littlewood maximal function. Rend. Mat. Appl. (7) 7, 1987, 273-279.

[13] Coifman, R., and G. Weiss: Analyse harmonique noncommutative sur certains espaces homogénes. - Lecture Notes in Math. 24, Springer-Verlag, Heidelberg, New York, 1971.

[14] Cruz-Uribe, D., SFO, A. Fiorenza, J. Martell, and C. Pérez: The boundedness of classical operators on variable $L^{p}$ spaces. - Ann. Acad. Sci. Fenn. Math. 31, 2006, 239-264.

[15] Cruz-Uribe, D., A. Fiorenza, and C. Neugebauer: The maximal function on variable $L^{p}$ spaces. - Ann. Acad. Sci. Fenn. Math. 28, 2003, 223-238.

[16] Defant, A.: Variants of the Maurey-Rosenthal theorem for quasi Köthe function spaces. Positivity 5, 2001, 153-175.

[17] Diening, L.: Maximal functions on generalized Lebesgue spaces $L^{p(\cdot)}$. - Math. Inequal. Appl. $7,2004,245-253$.

[18] Diening, L.: Riesz potential and Sobolev embeddings on generalized Lebesgue and Sobolev spaces $L^{p(\cdot)}$ and $W^{k, p(\cdot)}$. - Math. Nachr. 268, 2004, 31-43.

[19] Diening, L.: Maximal function on Orlicz-Musielak spaces and generalized Lebesgue space. Bull. Sci. Math. 129, 2005, 657-700.

[20] Diening, L., P. Hästö, and S. Roudenko: Function spaces of variable smoothness and integrability. - J. Funct. Anal. 256, 2009, 1731-1768.

[21] Diening, L., and M. RŮŽIČKA: Calderón-Zygmund operators on generalized Lebesgue space $L^{p(\cdot)}$ and problems related to fluid dynamics. - J. Reine Angew. Math. 563, 2003, 197-220.

[22] Frazier, M., and B. Jawerth: Decomposition of Besov spaces. - Indiana Univ. Math. J. 34, $1985,777-799$.

[23] Frazier, M., and B. Jawerth: The $\varphi$-transform and applications to distribution spaces. - In: Function spaces and applications (Lund, 1986), Lecture Notes in Math. 1302, Springer-Verlag, $1988,223-246$.

[24] Frazier, M., and B. Jawerth: A discrete transform and decomposition of distribution spaces. - J. Funct. Anal. 93, 1990, 34-170.

[25] Frazier, M., B. Jawerth, and G. Weiss: Littlewood-Paley theory and the study of function spaces. - CBMS Regional Conference Ser. 79, Amer. Math. Soc., 1991.

[26] FU, J., and J. XU: Characterizations of Morrey type Besov and Triebel-Lizorkin spaces with variable exponents. - J. Math. Anal. Appl. 381, 2011, 280-298.

[27] García-Cuerva, J.: Factorization of operators and weighted norm inequalities. - In: Nonlinear Analysis, Function Spaces and Applications, Proceedings of the Spring School held in Roudnice and Labem, Teubner, Leipzig, 1990, 5-41.

[28] García-Cuerva, J., and J. L. Rubio de Francia: Weighted norm inequalities and related topics. - North-Holland, 1985. 
[29] Giga, Y., and T. MiYakawa: Navier-Stokes flow in $\mathbf{R}^{3}$ with measures as initial vorticity and Morrey spaces. - Comm. Partial Differential Equations 14, 1989, 577-618.

[30] Grafakos, L.: Modern Fourier analysis. - Springer, second edition, 2009.

[31] Ho, K.-P.: Remarks on Littlewood-Paley analysis. - Canad. J. Math. 60, 2008, 1283-1305.

[32] Ho, K.-P.: Littlewood-Paley spaces. - Math. Scand. 108, 2011, 77-102.

[33] Ho, K.-P.: Characterization of $B M O$ by $A_{p}$ weights and $p$-convexity. - Hiroshima Math. J. 41, 2011, 153-165.

[34] Ho, K.-P.: Generalized Boyd's indices and applications. - Analysis (Munich) 32, 2012, 97-106.

[35] Ho, K.-P.: Atomic decompositions of weighted Hardy-Morrey Spaces. - Hokkaido Math. J. (to appear).

[36] Hudzik, H.: The problems of separability, duality, reflexivity and of comparison for generalized Orlicz-Sobolev spaces $W_{M}^{k}(\Omega)$. - Comment. Math. (Prace Mat.) 21, 1979, 315-324.

[37] Izuki, M.: Boundedness of commutators on Herz spaces with variable exponent. - Rend. Circ. Mat. Palermo (2) 59, 2010, 199-213.

[38] Kalita, E.: Dual Morrey spaces. - Dokl. Math. 58, 1998, 85-87.

[39] Kalton, N., N. Peck, and J. Roberts: An F-space sampler. - London Math. Soc. Lecture Note Ser. 89, Cambridge Univ. Press, 1984.

[40] Kato, T.: Strong solutions of the Navier-Stokes equation in Morrey spaces. - Boll. Soc. Brasil Math. 22, 1992, 127-155.

[41] Kokilashvili, V., and A. Meskhi: Boundedness of maximal and singular operators in Morrey spaces with variable exponent. - Armen. J. Math. 1, 2008, 18-28.

[42] Kokilashvili, V., and S. Samko: Singular integrals in weighted Lebesgue spaces with variable exponent. - Georgian Math. J. 10, 2003, 145-156.

[43] Komori, Y., and S. ShiraI: Weighted Morrey spaces and a singular integral operator. - Math. Nachr. 282, 2009, 219-231.

[44] KováčIK, O., and J. RÁKosník: On spaces $L^{p(\cdot)}$ and $W^{k, p(\cdot)}$. Czechoslovak Math. J. 41, $1991,592-618$.

[45] Krivine, J.-L.: Théorèmes de factorisation dans les espaces réticulés. - Séminaire MaureySchwartz 1973-74: Espaces $L^{p}$, applications radonifiantes et géométrie das espaces de Banach, Exp. Nos. 22 et 23, Centre de Math., École Polytechnique, Paris, 1974 (in French).

[46] Kufner, A., O. John, and S. FučIK: Function spaces. - Noordhoff International Publishing, 1977.

[47] Kurtz, D.: Littlewood-Paley and multiplier theorems on weighted $L^{p}$ spaces. - Trans. Amer. Math. Soc. 259, 1980, 235-254.

[48] Lindenstrauss, J., and L. Tzafriri: Classical Banach spaces I and II. - Springer, 1996.

[49] Leopold, H.-G.: On Besov spaces of variable order of differentiation. - Z. Anal. Anwend. 8, $1989,69-82$.

[50] Leopold, H.-G.: On function spaces of variable order of differentiation. - Forum Math. 3, 1991, 633-644.

[51] Mazzucato, A.: Besov-Morrey spaces: Function space theory and applications to non-linear PDE. - Trans. Amer. Math. Soc. 355, 2003, 1297-1364.

[52] Mazzuchto, A.: Decomposition of Besov-Morrey spaces. - In: Harmonic analysis at Mount Holyoke (South Hadley, MA, 2001), Contemp. Math. 320, Amer. Math. Soc., 2003, 279-294.

[53] Mizuhara, T.: Boundedness of some classical operators on generalized Morrey spaces. - In: Harmonic Analysis, ICM 90 Satellite Proceedings, Springer-Verlag, 1991, 183-189. 
[54] Morrey, C.: On the solutions of quasi-linear elliptic partial differential equations. - Trans. Amer. Math. Soc. 43, 1938, 126-166.

[55] Montgomery-Smith, S.: The Hardy operator and Boyd indices. Interaction between functional analysis, harmonic analysis, and probability. - Marcel Dekker, 1996.

[56] NAKAI, E.: Hardy-Littlewood maximal operator, singular integral operators and the Riesz potentials on generalized Morrey spaces. - Math. Nachr. 166, 1994, 95-104.

[57] Nekvinda, A.: Hardy-Littlewood maximal operator on $L^{p(x)}\left(\mathbf{R}^{n}\right)$. - Math. Inequal. Appl. 7, 2004, 255-265.

[58] Okada, S., W. Ricker, and E. Sánchez Pérez: Optimal domain and integral extension of operators. - Birkhäuser, Basel, 2008.

[59] Peetre, J.: On convolution operators leaving $L^{p, \lambda}$ space invariant. - Ann. Mat. Pura Appl. $72,1966,295-304$.

[60] Peetre, J.: On the theory of $\mathcal{L}_{p, \lambda}$ spaces. - J. Funct. Anal. 4, 1969, 71-87.

[61] Rajagopal K., and M. RŮŽIČKA; On the modeling of electrorheological materials. - Mech. Res. Comm. 23, 1996, 401-407.

[62] Rubio de Francia, J., F. Ruiz, and J. Torrea: Calderón-Zygmund theory for vectorvalued functions. - Adv. Math. 62, 1986, 7-48.

[63] RŮŽIČKA, M.: Electrorheological fluids: Modeling and mathematical theory. - Lecture Notes in Math. 1748, Springer-Verlag, Berlin, 2000.

[64] RŮŽIČKA, M.: Modeling, mathematical and numerical analysis of electrorheological fluids. Appl. Math. 49, 2004, 565-609.

[65] Sawano, Y., and H. Tanaka: Morrey spaces for non-doubling measures. - Acta Math. Sin. (Engl. Ser.) 21, 2005, 1535-1544.

[66] Sawano, Y., and H. Tanaka: Decompositions of Besov-Morrey spaces and Triebel-LizorkinMorrey spaces. - Math. Z. 257, 2007, 871-905.

[67] Sickel, W., D. Yang, and W. YuAn: Morrey and Campanato meet Besov, Lizorkin and Triebel. - Lecture Notes in Math. 2005, Springer, Berlin, 2010.

[68] Spanne, S.: Sur l'interpolation entre les espaces $\mathcal{L}_{k}^{p \Phi}$. - Ann. Sc. Norm. Super. Pisa 20, 1966, 625-648.

[69] Stampacchia, G.: $\mathcal{L}^{(p, \lambda)}$-spaces and interpolation. - Comm. Pure Appl. Math. 12, 1964, 293306.

[70] Stampacchia, G.: The spaces $\mathcal{L}^{(p, \lambda)}, N^{(p, \lambda)}$ and interpolation. - Ann. Sc. Norm. Super. Pisa 19, 1965, 443-462.

[71] TAng, L., and J. Xu: Some properties of Morrey type Besov-Triebel spaces. - Math. Nachr. 278, 2005, 904-917.

[72] TAYlor, M.: Analysis on Morrey spaces and applications to Navier-Stokes and other evolution equations. - Comm. Partial Differential Equations 17, 1992, 1407-1456.

[73] Triebel, H.: Theory of function spaces. - Monographs in Math. 78, Birkhäuser, 1983.

[74] Wang, H.: Decomposition for Morrey type Besov-Triebel spaces. - Math. Nachr. 282, 2009, $774-787$.

[75] Xu, J.-S.: Variable Besov and Triebel-Lizorkin spaces. - Ann. Acad. Sci. Fenn. Math. 33, 2008, 511-522.

[76] Zorko, C.: Morrey space. - Proc. Amer. Math. Soc. 98, 1986, 586-592. 